\title{
THE ATACAMA COSMOLOGY TELESCOPE: DYNAMICAL MASSES AND SCALING RELATIONS FOR A SAMPLE OF MASSIVE SUNYAEV-ZEL'DOVICH EFFECT SELECTED GALAXY CLUSTERS ${ }^{* \dagger}$
}

\author{
Cristóbal Sifón $^{1,2,21}$, Felipe Menanteau ${ }^{3,21}$, Matthew Hasselfield ${ }^{4}$, Tobias A. Marriage ${ }^{5}$ John P. Hughes $^{3,21}$, \\ L. Felipe Barrientos ${ }^{1}$, Jorge González ${ }^{1}$, Leopoldo Infante ${ }^{1}$, Graeme E. Addison ${ }^{6}$, Andrew J. BaKer ${ }^{3}$, Nick Battaglia ${ }^{7}$, \\ J. Richard Bond ${ }^{7}$, Devin Crichton ${ }^{5}$, Sudeep Das ${ }^{8}$, Mark J. Devlin ${ }^{9}$, JoAnna Dunkley ${ }^{6}$, Rolando Dünner ${ }^{1}$, \\ Megan B. Gralla ${ }^{5}$, Amir Hajian ${ }^{7}$, Matt Hilton ${ }^{10}$, Adam D. Hincks ${ }^{7,11}$, Arthur B. Kosowsky ${ }^{12}$, Danica Marsden $^{13}$, \\ Kavilan Moodley ${ }^{14}$, Michael D. Niemack ${ }^{15}$, Michael R. Nolta ${ }^{7}$, Lyman A. Page ${ }^{11}$, Bruce Partridge ${ }^{16}$, Erik D. Reese ${ }^{9}$, \\ Neelima Sehgal ${ }^{17}$, Jon Sievers ${ }^{7}$, David N. Spergel ${ }^{17}$, Suzanne T. Staggs ${ }^{11}$, Robert J. Thornton ${ }^{9,18}$, \\ Hy Trac ${ }^{19}$, AND EDWARd J. Wollack ${ }^{20}$ \\ ${ }^{1}$ Departamento de Astronomía y Astrofísica, Facultad de Física, Pontificia Universidad Católica de Chile, Casilla 306, Santiago 22, Chile \\ ${ }^{2}$ Leiden Observatory, Leiden University, P.O. Box 9513, NL-2300 RA Leiden, The Netherlands \\ ${ }^{3}$ Department of Physics and Astronomy, Rutgers University, 136 Frelinghuysen Road, Piscataway, NJ 08854, USA \\ ${ }^{4}$ Department of Physics and Astronomy, University of British Columbia, Vancouver, BC V6T 1Z4, Canada \\ ${ }^{5}$ Department of Physics and Astronomy, The Johns Hopkins University, Baltimore, MD 21218-2686, USA \\ ${ }^{6}$ Sub-department of Astrophysics, University of Oxford, Denys Wilkinson Building, Keble Road, Oxford OX1 3RH, UK \\ ${ }^{7}$ Canadian Institute for Theoretical Astrophysics, University of Toronto, Toronto, ON M5S 3H8, Canada \\ ${ }^{8}$ Berkeley Center for Cosmological Physics, LBL and Department of Physics, University of California, Berkeley, CA 94720, USA \\ ${ }^{9}$ Department of Physics and Astronomy, University of Pennsylvania, 209 South 33rd Street, Philadelphia, PA 19104, USA \\ ${ }^{10}$ School of Physics and Astronomy, University of Nottingham, University Park, Nottingham, NG7 2RD, UK \\ ${ }^{11}$ Joseph Henry Laboratories of Physics, Jadwin Hall, Princeton University, Princeton, NJ 08544, USA \\ ${ }^{12}$ Physics and Astronomy Department, University of Pittsburgh, 100 Allen Hall, 3941 O'Hara Street, Pittsburgh, PA 15260, USA \\ ${ }^{13}$ Department of Physics, University of California-Santa Barbara, Santa Barbara, CA 93106-9530, USA \\ ${ }^{14}$ University of KwaZulu-Natal, Astrophysics and Cosmology Research Unit, School of Mathematical Sciences, Durban, 4041, South Africa \\ ${ }^{15}$ NIST Quantum Devices Group, 325 Broadway Mailcode 817.03, Boulder, CO 80305, USA \\ ${ }^{16}$ Department of Physics and Astronomy, Haverford College, Haverford, PA 19041, USA \\ ${ }^{17}$ Department of Astrophysical Sciences, Peyton Hall, Princeton University, Princeton, NJ 08544, USA \\ ${ }^{18}$ Department of Physics, West Chester University, West Chester, PA 19383, USA \\ ${ }^{19}$ Department of Physics, Carnegie Mellon University, Pittsburgh, PA 15213, USA \\ ${ }^{20}$ NASA/Goddard Space Flight Center, Greenbelt, MD 20771, USA \\ Received 2012 January 4; accepted 2013 May 22; published 2013 July 2
}

\begin{abstract}
We present the first dynamical mass estimates and scaling relations for a sample of Sunyaev-Zel'dovich effect (SZE) selected galaxy clusters. The sample consists of 16 massive clusters detected with the Atacama Cosmology Telescope (ACT) over a $455 \mathrm{deg}^{2}$ area of the southern sky. Deep multi-object spectroscopic observations were taken to secure intermediate-resolution $(R \sim 700-800)$ spectra and redshifts for $\approx 60$ member galaxies on average per cluster. The dynamical masses $M_{200 c}$ of the clusters have been calculated using simulation-based scaling relations between velocity dispersion and mass. The sample has a median redshift $z=0.50$ and a median mass $M_{200 c} \simeq 12 \times 10^{14} h_{70}^{-1} M_{\odot}$ with a lower limit $M_{200 c} \simeq 6 \times 10^{14} h_{70}^{-1} M_{\odot}$, consistent with the expectations for the ACT southern sky survey. These masses are compared to the ACT SZE properties of the sample, specifically, the match-filtered central SZE amplitude $\tilde{y_{0}}$, the central Compton parameter $y_{0}$, and the integrated Compton signal $Y_{200 c}$, which we use to derive SZE-mass scaling relations. All SZE estimators correlate with dynamical mass with low intrinsic scatter $(\lesssim 20 \%)$, in agreement with numerical simulations. We explore the effects of various systematic effects on these scaling relations, including the correlation between observables and the influence of dynamically disturbed clusters. Using the three-dimensional information available, we divide the sample into relaxed and disturbed clusters and find that $\sim 50 \%$ of the clusters are disturbed. There are hints that disturbed systems might bias the scaling relations, but given the current sample sizes, these differences are not significant; further studies including more clusters are required to assess the impact of these clusters on the scaling relations.
\end{abstract}

Key words: cosmic background radiation - cosmology: observations - galaxies: clusters: general - galaxies: distances and redshifts

Online-only material: color figures, machine-readable table

\footnotetext{
* Based in part on observations collected at the European Organisation for Astronomical Research in the Southern Hemisphere, Chile, under programs 084.A-0577 and 086.A-0425.

$\dagger$ Based in part on observations obtained at the Gemini Observatory, which is operated by the Association of Universities for Research in Astronomy, Inc., under a cooperative agreement with the NSF on behalf of the Gemini partnership: the National Science Foundation (United States), the Science and Technology Facilities Council (United Kingdom), the National Research Council (Canada), CONICYT (Chile), the Australian Research Council (Australia), Ministério da Ciência e Tecnologia (Brazil), and Ministerio de Ciencia, Tecnología e Innovación Productiva (Argentina).

${ }^{21}$ Visiting Astronomer, Gemini South Observatory.
}

\section{INTRODUCTION}

Studies of clusters of galaxies have had a wide impact on our understanding of galaxy formation and cosmology (see Voit 2005, for a review). They are a unique laboratory for studying the effects of the environment (high density, gas pressure, collisions, etc.) on galaxy evolution (Butcher \& Oemler 1984; Balogh et al. 1999; Hansen et al. 2009). At the same time, number counts of galaxy clusters, sensitive to the amplitude of matter fluctuations, can provide constraints on various 
cosmological parameters (Bahcall \& Fan 1998; Evrard et al. 2002; Vikhlinin et al. 2009; Mantz et al. 2010b, 2010c; Rozo et al. 2010). An accurate determination of the latter requires that we know the mass and redshift distributions of clusters with good precision.

The Sunyaev-Zel'dovich effect (SZE; Zel'dovich \& Sunyaev 1969; Sunyaev \& Zel'dovich 1970) is a distortion in the cosmic microwave background (CMB) temperature produced by inverse-Compton scattering of CMB photons as they interact with the hot electrons of the intracluster medium (ICM) of a galaxy cluster. Its surface brightness is independent of redshift, and its strength is proportional to the line-of-sight (LOS) column density times the electron temperature. The SZE is a powerful tool for detecting massive clusters to high redshifts (see, e.g., the reviews by Birkinshaw 1999; Carlstrom et al. 2002).

Early measurements of the SZE were achieved with targeted observations of known clusters. These revealed the power of SZE studies, reaching from gas physics and inner structure of clusters (Grego et al. 2001; Benson et al. 2004) to cosmological parameters such as the Hubble constant (Birkinshaw et al. 1991; Hughes \& Birkinshaw 1998) and the energy density of matter in the universe, $\Omega_{M}$ (Grego et al. 2001). Large SZE surveys over cosmologically significant areas of the sky have recently come to fruition as the Atacama Cosmology Telescope (ACT; Fowler et al. 2007; Swetz et al. 2011) and the South Pole Telescope (SPT; Carlstrom et al. 2011) have begun scanning large areas of the sky at millimeter wavelengths. The Planck satellite (Tauber et al. 2010) is conducting an all-sky survey and has recently released the first all-sky sample of SZE-selected galaxy clusters (Planck Collaboration 2011a). The first cluster detections with ACT and SPT are presented in Hincks et al. (2010) and Staniszewski et al. (2009), respectively.

The rapidly growing SZE cluster samples have the potential to place strong constraints on cosmological parameters (e.g., Battye \& Weller 2003). Both numerical simulations (Springel et al. 2001a; da Silva et al. 2004; Motl et al. 2005; Nagai 2006; Battaglia et al. 2012) and analytical studies (Reid \& Spergel 2006; Ashfordi 2008; Shaw et al. 2008) suggest a tight correlation between cluster mass and SZE signal. On the other hand, biased mass estimates can have a large impact on cosmological parameter determination (e.g., Francis et al. 2005). By limiting their study to the high-significance clusters, Sehgal et al. (2011) have shown the power of the ACT sample in constraining cosmological parameters, particularly the dark energy equation-of-state parameter $w$ and the rms mass fluctuations on a scale of $8 h^{-1} \mathrm{Mpc}, \sigma_{8}$. Likewise, Vanderlinde et al. (2010) have used SPT data to set cosmological constraints, with similar findings. They have also shown that these improvements can be achieved only in the presence of a well-calibrated scaling relation between mass and SZE signal. To assess the scaling of SZE signal with mass, independent means of measuring the mass are crucial.

Benson et al. (2013) used X-ray observations in combination with SZE measurements to derive an empirical scaling relation between mass and SZE signal. This allowed them to confirm that SZE-selected samples of clusters yield significant improvements when added to other data sets to constrain cosmological parameters. While X-ray observations have proven to be an effective way of measuring cluster masses and have been exploited to characterize the SZE signal (LaRoque et al. 2006; Bonamente et al. 2008; Andersson et al. 2011; Melin et al. 2011; Planck Collaboration $2011 \mathrm{~b}$ ), they do not provide truly independent mass estimates from SZE measurements, since both rely on the prop- erties of the gas in the ICM and should be affected by similar physics.

The velocity dispersion of cluster member galaxies is one of the most widely used methods for constraining cluster mass and is independent of the properties of the gas in the ICM. It takes into account the galaxy distribution and relies, to some extent, on the assumption that the clusters are relaxed (i.e., virialized). Until recently, however, mass measurements to independently calibrate the SZE signal with mass have come from optical richness (Menanteau \& Hughes 2009; High et al. 2010; Menanteau et al. 2010b; Planck Collaboration 2011c) and lensing analyses (Sealfon et al. 2006; Umetsu et al. 2009; Marrone et al. 2012). Hand et al. (2011) presented stacked ACT data in the directions of luminous red galaxies from the Sloan Digital Sky Survey (SDSS) Data Release 7 (DR7; Abazajian et al. 2009) using optical luminosity-based masses. This approach allowed them to probe the SZE signal from lower mass systems than otherwise possible.

Rines et al. (2010) presented the first statistical comparison between dynamically estimated masses and integrated SZE signal from a sample of 15 nearby $(z<0.3)$ galaxy clusters, showing that masses thus determined and the integrated SZE flux are correlated at the $\approx 99 \%$ confidence level. Furthermore, they estimate that the significance is higher than that of the correlation between SZE and weak-lensing masses from Marrone et al. (2009), probably because of the smaller apertures used in the latter study. However, since their sample was not homogeneously selected, Rines et al. (2010) do not account for observational biases in their sample and do not report a formal scaling relation between mass and SZE flux.

In this work, we present spectroscopic redshifts and-for the first time-dynamical masses of a sample of clusters of galaxies selected with the SZE. These clusters were observed by ACT in its 2008 southern sky survey at $148 \mathrm{GHz}$ (Marriage et al. 2011a) and were optically confirmed by Menanteau et al. (2010a). We use a variety of SZE diagnostics to assess the scaling with dynamical mass and thus present the first robust scaling relations between dynamical masses and SZE signal for a sample of SZE-selected clusters.

Throughout this work we use a flat $\Lambda$ CDM cosmology consistent with WMAP-7 data (Komatsu et al. 2011), with $\Omega_{\Lambda}=$ $0.73, \Omega_{M}=0.27$, and $H_{0}=70 h_{70} \mathrm{~km} \mathrm{~s}^{-1} \mathrm{Mpc}^{-1}$. Masses and integrated SZE signals are estimated within a radius $r_{200 c}$ which encloses a density 200 times the critical density of the universe at the redshift of the cluster, $\rho_{c}(z)=3 H^{2}(z) / 8 \pi G$. All quoted errors are $68 \%$ confidence intervals unless otherwise stated.

\section{OBSERVATIONS}

\subsection{ACT SZE Observations}

ACT is a $6 \mathrm{~m}$ off-axis Gregorian telescope operating at an altitude of $5200 \mathrm{~m}$ in the Atacama Desert in Chile, designed to observe the CMB at arcminute-scale resolution. It has three 1024-element arrays of transition edge sensors operating at 148, 218, and $277 \mathrm{GHz}$. ACT surveyed two regions of the sky, of which $755 \mathrm{deg}^{2}$ have been used for cluster studies (Marriage et al. 2011b; Hasselfield et al. 2013). The processes of cluster detection and extraction are thoroughly described in Marriage et al. (2011a), and references therein. In short, the maps are match-filtered and convolved with a beta-model profile with $\beta=0.86$ with varying core radius $\theta_{c}$ from 0.25 to 4.0 . Cluster signal-to-noise ratio $(\mathrm{S} / \mathrm{N})$ is measured as the maximum $\mathrm{S} / \mathrm{N}$ from this set of filtered maps. 
We report on a large spectroscopic follow-up campaign of an ACT $148 \mathrm{GHz}$ cluster sample, which was obtained from a $455 \mathrm{deg}^{2}$ survey of the southern sky. The survey is roughly bounded by right ascensions $00^{\mathrm{h}} 12^{\mathrm{m}}$ and $07^{\mathrm{h}} 08^{\mathrm{m}}$ and declinations $-56^{\circ} 11^{\prime}$ and $-49^{\circ} 00^{\prime}$. For further details on the ACT observations, map making, data reduction, and cluster detection procedure, see Fowler et al. (2010), Marriage et al. (2011a), and Dünner et al. (2013).

\subsubsection{The Cluster Sample}

In this study we consider a total of 19 clusters, spanning a wide range in mass and redshift. We focus, in particular, on the subsample of 16 clusters that were detected by ACT through their SZ signal. This subsample contains 15 systems that were detected by ACT in the 2008 single-season maps (Marriage et al. 2011a) and confirmed optically on $4 \mathrm{~m}$ class telescopes (Menanteau et al. 2010a), plus one additional cluster (ACTCL J0521-5104) detected in the new analysis of multi-season maps. This latter cluster was initially targeted for spectroscopic follow-up based on its optical richness alone (Menanteau et al. 2010 b). The 16 clusters were selected based on a redshift cut of $z_{\text {phot }}>0.35$ and were all discovered with the SZE, with the exceptions of ACT-CL J0330-5227 (X-rays; Werner et al. 2007) and ACT-CL J0521-5104 (optical; Menanteau et al. 2010b). ACT-CL J0330-5227 is located 12' northeast (NE) of A3128 ( $z=0.06$; Colless \& Hewett 1987; Katgert et al. 1996), but Werner et al. (2007) found it to be an unrelated, background cluster at $z=0.44$ based on the observed energy of the Fe K X-ray emission line using XMM-Newton observations and the optical spectrum of the brightest cluster galaxy (BCG). Hincks et al. (2010) have shown that the observed SZE signal is clearly related to the background cluster. Four clusters were initially reported by SPT (Staniszewski et al. 2009; Vanderlinde et al. 2010, see Section 7.1) and studied optically by Menanteau et al. (2010b). ACT-CL J0546-5345 is the only cluster with a dynamical mass estimate prior to this study (Brodwin et al. 2010; see Section 7.1.9). Recently, Hilton et al. (2013) presented a study of the stellar content of 14 of these 16 clusters from Spitzer observations.

Thus, of the 16 SZE-detected clusters reported here, 10 are newly discovered by ACT. Menanteau et al. (2010a) confirmed them as clusters with a BCG and an accompanying red sequence of galaxies and studied their X-ray properties from archival ROSAT data for the 15 clusters, plus Chandra and/or XMMNewton data when available. The clusters cover the range $\sim(1-30) \times 10^{44} \mathrm{erg} \mathrm{s}^{-1}$ in X-ray luminosity as measured in the $0.1-2.4 \mathrm{keV}$ band. Photometric redshifts were estimated for these 15 clusters by Menanteau et al. (2010a). The spectroscopic redshift range covered by the sample is $0.28<z<1.07$ with a median redshift $z=0.50$.

Additionally, we report on three optically selected, highrichness galaxy clusters from the Southern Cosmology Survey (SCS; Menanteau et al. 2010b). These clusters were part of our 2009B follow-up observations before the ACT maps were available for cluster detection, and were not detected by ACT. They are briefly discussed in Section 7.2.

\subsubsection{Cluster SZE Measurements}

To characterize the SZE produced by each cluster (in the $148 \mathrm{GHz}$ band) we study three different estimators. These values are listed in Table 1 and are all measured using multi-season (2008-2010) ACT data.
Table 1

ACT-SZE Measurements of Clusters

\begin{tabular}{lcccc}
\hline \hline Cluster & $z$ & $\begin{array}{c}\tilde{y}_{0}^{\mathrm{a}} \\
\left(10^{-4}\right)\end{array}$ & $\begin{array}{c}y_{0}{ }^{\mathrm{b}} \\
\left(10^{-4}\right)\end{array}$ & $\begin{array}{c}Y_{200 c}{ }^{\mathrm{c}} \\
\left(10^{-10}\right)\end{array}$ \\
\hline ACT-CL J0102-4915 & 0.870 & $3.51 \pm 0.43$ & $5.66 \pm 0.62$ & $1.47 \pm 0.18$ \\
ACT-CL J0215-5212 & 0.480 & $0.78 \pm 0.18$ & $1.10 \pm 0.25$ & $0.37 \pm 0.10$ \\
ACT-CL J0232-5257 & 0.556 & $0.60 \pm 0.17$ & $0.91 \pm 0.28$ & $0.28 \pm 0.07$ \\
ACT-CL J0235-5121 & 0.278 & $0.99 \pm 0.19$ & $1.03 \pm 0.21$ & $0.97 \pm 0.20$ \\
ACT-CL J0237-4939 & 0.334 & $0.93 \pm 0.26$ & $0.94 \pm 0.32$ & $1.07 \pm 0.31$ \\
ACT-CL J0304-4921 & 0.392 & $1.59 \pm 0.31$ & $1.68 \pm 0.37$ & $1.05 \pm 0.25$ \\
ACT-CL J0330-5227 & 0.442 & $1.25 \pm 0.18$ & $1.61 \pm 0.21$ & $0.90 \pm 0.13$ \\
ACT-CL J0346-5438 & 0.530 & $1.05 \pm 0.22$ & $1.48 \pm 0.30$ & $0.46 \pm 0.11$ \\
ACT-CL J0438-5419 & 0.421 & $1.63 \pm 0.13$ & $2.06 \pm 0.15$ & $1.14 \pm 0.10$ \\
ACT-CL J0509-5341 & 0.461 & $0.82 \pm 0.14$ & $0.59 \pm 0.19$ & $0.12 \pm 0.05$ \\
ACT-CL J0521-5104 & 0.675 & $0.72 \pm 0.16$ & $1.31 \pm 0.25$ & $0.28 \pm 0.07$ \\
ACT-CL J0528-5259 & 0.768 & $0.49 \pm 0.13$ & $1.03 \pm 0.27$ & $0.10 \pm 0.03$ \\
ACT-CL J0546-5345 & 1.066 & $0.92 \pm 0.14$ & $2.36 \pm 0.30$ & $0.26 \pm 0.03$ \\
ACT-CL J0559-5249 & 0.609 & $0.90 \pm 0.14$ & $1.51 \pm 0.20$ & $0.51 \pm 0.05$ \\
ACT-CL J0616-5227 & 0.684 & $1.00 \pm 0.15$ & $1.80 \pm 0.22$ & $0.47 \pm 0.05$ \\
ACT-CL J0707-5522 & 0.296 & $0.52 \pm 0.21$ & $0.51 \pm 0.22$ & $0.57 \pm 0.13$ \\
\hline
\end{tabular}

Notes. Redshifts are listed for reference. See Table 3 for details.

a Central match-filtered amplitude of the SZE, measured using the A10 profile with an FWHM of $2^{\prime}$. See Hasselfield et al. (2013).

b Projected central Compton parameter assuming the A10 profile. See Hasselfield et al. (2013).

c Spherically integrated Compton amplitude within $r_{200 c}$ assuming the A10 profile. See Section 2.1.2.

The first estimator, $\tilde{y_{0}}$, corresponds to the central matchfiltered SZE amplitude. A detailed description of the procedure is given in Hasselfield et al. (2013, see their Section 2.2) but is outlined here. The ACT maps are passed through a matched filter to extract the amplitude of the temperature decrement of clusters modeled with the universal pressure profile of Arnaud et al. (2010)—-hereafter "the A10 profile"-with a fixed scale $\theta_{\text {FWHM }}=2^{\prime}$. This scale is related to the more usual parameterization of the characteristic scale by $\theta_{500 c}=$ $2.94 \theta_{\text {FWHM }}$ given the best-fit concentration parameter from Arnaud et al. (2010), $c_{500 c}=1.177$. Although the filter accounts for the effects of the beam in the signal template, its normalization is chosen to return the central decrement of the corresponding unconvolved cluster signal. The central temperature decrement is scaled to a central Compton parameter using the standard non-relativistic SZE frequency dependence (Sunyaev \& Zel'dovich 1980).

Using $\widetilde{y_{0}}$ and assuming that the pressure profile follows the (mass dependent) A10 profile, one can estimate what the actual central Compton parameter should be. While this measure carries some assumptions about the physics of the cluster and the relation between pressure and mass (i.e., it is modeldependent), it is completely independent from the reported dynamical masses and it is thus still useful to compare both quantities. The central Compton parameter is referred to as $y_{0}$, as usual. A more detailed discussion about $\tilde{y_{0}}$ and $y_{0}$ can be found in Hasselfield et al. (2013).

Our third measurement is the integrated Compton signal. Large integration areas tend to give measurements that are more robust against the effects of cluster physics such as active galactic nucleus (AGN) feedback (Motl et al. 2005; Nagai 2006; Reid \& Spergel 2006), and to projection effects (Shaw et al. 2008). Dynamical masses are usually measured at $r_{200 c}$ (see Section 4.1), providing therefore a measurement of the size of the cluster. Since the parameterization of the A10 profile is 
Table 2

Spectroscopic Observation Details

\begin{tabular}{|c|c|c|c|c|c|c|c|c|}
\hline Run & Semester & PI & Tel./Inst. & Program ID & Mode & Grating & Hours & $N_{\mathrm{cl}}$ \\
\hline 1 & 2009B & Infante & VLT/FORS2 & 084.A-0577 & Service & GRIS 300I+11 & 14 & 3 \\
\hline 2 & 2009B & Barrientos & Gemini-S/GMOS & GS-2009B-Q-2 & Service & R400_G5325 & 20 & 4 \\
\hline 3 & 2010B & Infante & VLT/FORS2 & 086.A-0425 & Service & GRIS 300I+11 & 15 & 2 \\
\hline 4 & 2010B & Barrientos/Menanteau ${ }^{\mathrm{a}}$ & Gemini-S/GMOS & GS-2010B-C-2 & Classical & R400_G5325 & 40 & 10 \\
\hline
\end{tabular}

Notes. $N_{\mathrm{cl}}$ is the number of clusters observed in each run. Each cluster was fully observed in one run.

a Joint Chile/US proposal.

given in terms of quantities measured at $r_{500 c}$, we convert values from $r_{200 c}$ to $r_{500 c}$ using a Navarro et al. (1995) profile (hereafter NFW profile) and the mass-concentration relation of Duffy et al. (2008). Combined with the dynamical information, this sets the scale of the filter through $\theta_{500 c}$. The filtering then returns the total integrated profile out to the virial radius, which is scaled to $r_{200 c}$ using the prescription of Arnaud et al. (2010). We refer to these spherical SZE measurements within $r_{200 c}$ as $Y_{200 c}$ hereafter. We estimate the covariance between $Y_{200 c}$ and $M_{200 c}$ by measuring $Y(<r)$ from the maps at different radii around $r_{200 c}$ for each cluster; the dynamical mass is re-scaled assuming a spherical cluster. This covariance is included in the determination of the scaling relations (see Sections 5.2 and 6.4).

\subsection{Optical Spectroscopy}

The spectroscopic observations were carried out in two semesters, 2009B and 2010B. Each semester was split into two observing runs, one with FORS2 at the Very Large Telescope (VLT; Appenzeller et al. 1998) and one with GMOS at Gemini South (Hook et al. 2004), both telescopes located in Chile. The details of each observing run are listed in Table 2. In total, we had 89 hr of observation, during which we collected multi-object spectroscopy for 19 clusters.

Targets were selected by a two-step process. First, a photometric redshift-selected catalog was constructed, including galaxies within \pm 0.1 of the redshift of the BCG. Within this catalog, galaxies were visually selected based on their gri colors, with preference given to bright galaxies. All our spectroscopic observations cover the wavelength range $\sim 4000-8000 \AA$. In this range, several spectral features are observable at the median photometric redshift of 0.54 (Menanteau et al. 2010a). These are mainly the $\mathrm{Ca}$ II $\mathrm{K}-\mathrm{H}$ absorption doublet (at a restframe wavelength $\lambda_{0} \sim 3950 \AA$ ), which is the spectral signature of elliptical galaxies, plus other absorption lines such as the $G$ band $\left(\lambda_{0}=4300 \AA\right), \mathrm{H} \beta\left(\lambda_{0}=4861 \AA\right)$, and the $\mathrm{Mg}$ II triplet $\left(\lambda_{0} \sim 5175 \AA\right)$, plus the [O II] emission line at rest-frame $\lambda_{0}=3727 \AA$. The Na I absorption doublet $\left(\lambda_{0} \sim 5892 \AA\right)$ is also observable in the low- $z$ clusters.

\subsubsection{VLT-FORS2 Observations}

The FORS2-2009B observations (Run 1) were aimed at newly SZE-detected clusters regarded as "secure" candidates detected with ACT in 2008. These clusters had already been reported as SZE detections by Staniszewski et al. (2009) and their physical properties characterized in Menanteau \& Hughes (2009).

Run 3 was mostly focused on getting detailed information for ACT-CL J0102-4915 ("El Gordo"; Menanteau et al. 2012), which was detected as the largest decrement in the ACT maps. ACT-CL J0559-5249 was also included in this run.

Runs 1 and 3 were executed in Service Mode in semesters 2009B and 2010B, respectively. The instrument setup in both runs was the same, using the GRIS $300 \mathrm{I}+11$ grism and $1^{\prime \prime}$ wide slits, which provides a resolving power $R=660$ at $\lambda=8600 \AA$. A total of 18 FORS2/MXU masks were observed for the five clusters. Each mask was observed for 40 minutes, which we estimated to be the best compromise between maximizing $\mathrm{S} / \mathrm{N}$ and number of masks.

FORS2 has a field of view of $6.8 \times 6.8$ in the standard resolution setup, which corresponds to a width of $2517 h_{70}^{-1} \mathrm{kpc}$ at $z=0.5$.

\subsubsection{Gemini-GMOS Observations}

The GMOS-2009B observations (Run 2) were aimed at four optically selected clusters from the SCS whose richness-based mass estimates suggested that they would be detected by ACT in the SZE survey (Menanteau et al. 2010b). However, as mentioned above, only one object was in fact detected by ACT (ACT-CL J0521-5104); the other three are discussed in Section 7.2. The total integration time per mask was $3600 \mathrm{~s}(2 \times$ $1800 \mathrm{~s})$. Two exposures at slightly different central wavelengths per mask were required to cover the two 37-pixel gaps between the CCDs which run across the dispersion axis.

Targets for Run 4 (GMOS-2010B) were selected from the sample of clusters newly discovered by ACT presented in Marriage et al. (2011a) and optically confirmed by Menanteau et al. (2010a). Run 4 was the only one executed in Classical Mode, during five consecutive nights (December 6-10), all with clear, photometric conditions and seeing $\lesssim 0$.'8. Based on our experience in Run 2, we decided to reduce the integration time to $2400 \mathrm{~s}(2 \times 1200 \mathrm{~s})$ for each mask during Run 4 . This, coupled with a $20 \%$ higher efficiency than Queue Mode, allowed us to observe a larger number of masks (and clusters) while still obtaining the necessary $\mathrm{S} / \mathrm{N}$ in the relevant spectral lines.

In both GMOS runs we used the R400_G5325 grating and 1" wide slits, providing a resolving power of $R \sim 800$ with a $2 \times 2$ binning at $\lambda \sim 7000 \AA$. In the standard setup GMOS has a field of view of $5.5 \times 5.5\left(2036 h_{70}^{-1} \mathrm{kpc}\right.$ at $\left.z=0.5\right)$.

\subsubsection{Data Reduction}

We have developed reduction pipelines both for the FORS2 and GMOS data, based on the existing software by ESO and Gemini, respectively, which work with IRAF/PyRAF. ${ }^{22}$ Cosmic rays are removed using L.A. Cosmic (van Dokkum 2001) with a detection limit of $4.5 \sigma$. The wavelength calibrations were done using CuAr lamps in the case of GMOS data and HeAr lamps for VLT data. The sky is subtracted from each spectrum using a constant value determined locally within each slitlet. In the case of GMOS data, the individual exposures are co-added at this point. Finally, the one-dimensional (1D) spectra are extracted

22 The pipeline used to reduce GMOS data—dubbed "pygmos"-is available at http://www.strw.leidenuniv.nl/ sifon/pygmos/. 


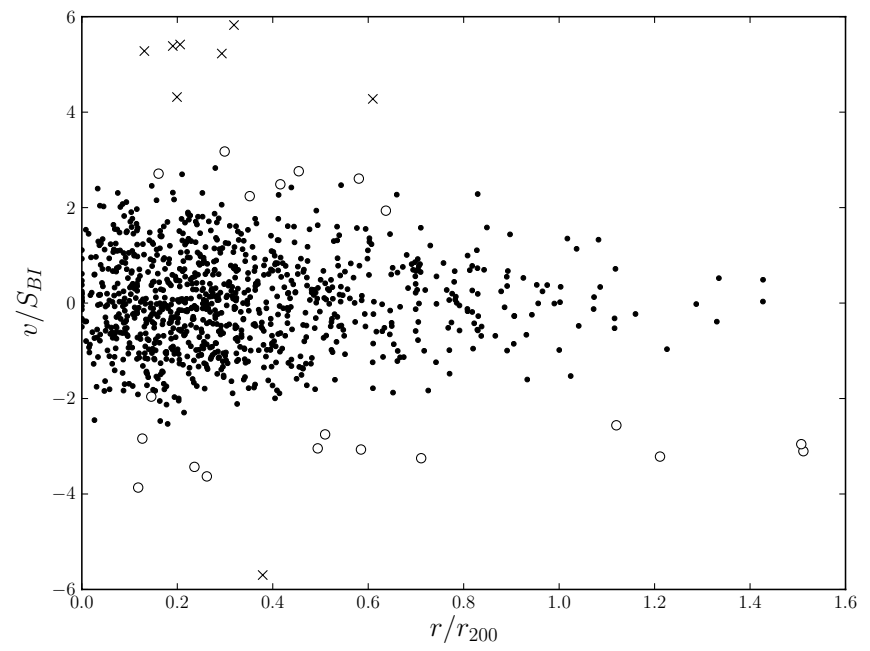

Figure 1. "Stacked" result of the shifting gapper method of member selection, showing the galaxies in all 16 SZE-detected clusters. The horizontal axis shows the cluster-centric distance normalized by $r_{200 c}$ for each cluster and the vertical axis shows the peculiar velocity of each galaxy, normalized by the velocity dispersion of the corresponding cluster. Black dots show member galaxies, open circles show galaxies rejected by the method, and crosses show galaxies with peculiar velocities larger than $4000 \mathrm{~km} \mathrm{~s}^{-1}$. Galaxies with peculiar velocities larger than $6 S_{\mathrm{BI}}$ are not shown for clarity.

from each slit and matched with the input photometric catalogs used to generate the masks.

\section{ANALYSIS AND RESULTS}

\subsection{Galaxy Redshifts}

Galaxy redshifts are measured by cross-correlating the spectra with galaxy spectral templates of the SDSS DR7 using the RVSAO/XCSAO package for IRAF (Kurtz \& Mink 1998); the spectral features in each spectrum have been confirmed with the $2 \mathrm{D}$ spectra by visual inspection. We have been able to estimate reliable redshifts for $\sim 1200$ galaxies which comprise $\sim 80 \%$ of all targeted objects.

The median rms in the wavelength calibration is $\sim 0.3 \AA$ and is similar for both instruments. At a central wavelength of $6000 \AA$, this corresponds to a velocity uncertainty of $15 \mathrm{~km} \mathrm{~s}^{-1}$, which is within the errors of the cross-correlation velocity. In particular, the latter is typically $\Delta(c z) \sim 40-80 \mathrm{~km} \mathrm{~s}^{-1}$, as calculated by RVSAO. It has been established experimentally that the true cross-correlation errors are larger than those reported by RVSAO, by a factor $\sim 1.7$ (e.g., Quintana et al. 2000), strengthening the point that the calibration errors are well within the velocity measurement errors.

We have included the member catalog for ACT-CL J0546-5345 published by Brodwin et al. (2010). Seven galaxies have been observed both by Brodwin et al. (2010) and by us; all redshifts are consistent within $2 \sigma$. We therefore use our measurements for those galaxies in the following analysis.

\subsection{Cluster Redshifts and Velocity Dispersions}

It is of great importance to correctly determine cluster membership to avoid a biased measurement of the velocity dispersion (Beers et al. 1991). This is a complicated problem and many methods have been developed to handle it. In this analysis, membership of galaxies to a cluster is determined by applying a cut in (rest frame) velocity space of $4000 \mathrm{~km} \mathrm{~s}^{-1}$, and then applying the shifting gapper method (Fadda et al. 1996).

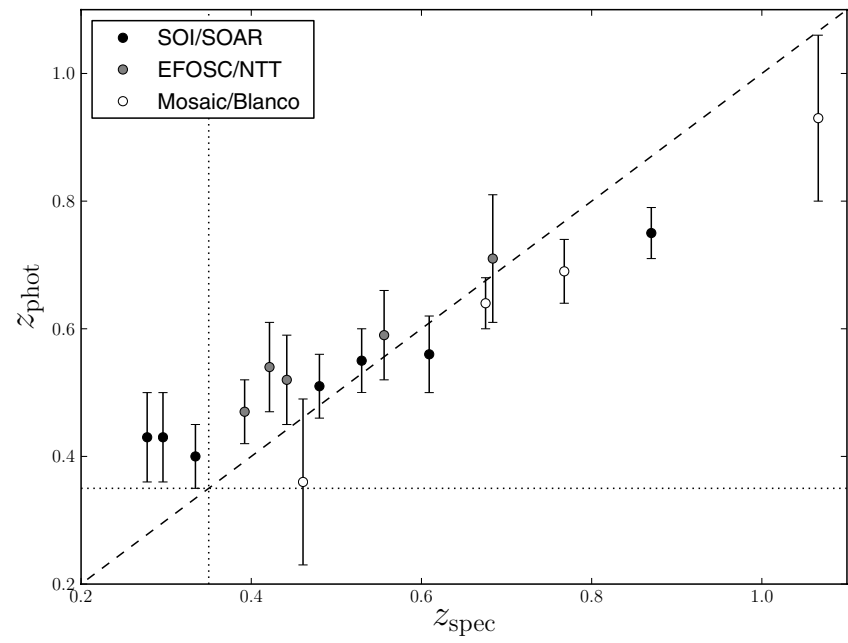

Figure 2. Comparison between spectroscopic redshifts from this work and initial gri photometric redshift estimates from Menanteau et al. (2010a). The instrument and telescope with which each cluster was observed are identified in the legend. The dashed line shows $z_{\text {phot }}=z_{\text {spec }}$. The dotted horizontal line shows the sample selection cut, $z_{\text {phot }}=0.35$, and the dotted vertical line shows the corresponding $z_{\mathrm{spec}}=0.35$.

To do this, we define annular bins around the BCG, each of which has at least 15 galaxies and radial width $\geqslant 250 h_{70}^{-1} \mathrm{kpc}$. We consider the histogram of velocities of member galaxies within each bin. We assume the profile is symmetric, and identify the main body of galaxies as those whose velocity is bounded by gaps of $\geqslant 500 \mathrm{~km} \mathrm{~s}^{-1}$. Following Katgert et al. (1996) and Fadda et al. (1996), galaxies separated from the main body by $\geqslant 1000 \mathrm{~km} \mathrm{~s}^{-1}$ are considered interlopers and are removed. The selection method is iterated until the number of members is stable. This usually happens after the second iteration. A total of 948 galaxies ( $\sim 65 \%$ of all targets) have been selected as cluster members. Most of these galaxies show the spectral signatures of elliptical galaxies and do not have emission lines, and only a few emission-line galaxies belong to clusters (see Section 4.3). The galaxies remaining at this point are considered members of the cluster. Figure 1 shows the "stacked" result of this method, with members as solid dots. The values have been normalized to allow for direct comparison of all clusters. We have explored systematic effects coming from the member selection method by changing the width of the bins, the number of galaxies per bin, and the size of either gap in the shifting gapper. Varying these parameters yields results that are consistent with the reported velocity dispersions.

We use the biweight estimators of location (hereafter $z_{\mathrm{BI}}$; Beers et al. 1990) for the redshift of the cluster and scale, $S_{\mathrm{BI}}$, for the velocity dispersion. All errors have been estimated with the bootstrap resampling technique with 5000 iterations. The redshifts of the clusters are presented in Figure 2, where they are compared to the photometric redshifts of Menanteau et al. (2010a). The median redshift of the sample is $z=0.50$. The slightly biased photometric redshifts apparent in Figure 2 are mainly due to two factors: the lack of a well-characterized filter response function for the telescopes involved in the imaging follow-up and the use of only three to four filters for the determination of photometric redshifts (Menanteau et al. 2010a).

Danese et al. (1980) showed that the observational errors on the redshifts of galaxies introduce a bias in the measured velocity dispersion. However, for a cluster of $M \sim 10^{15} M_{\odot}$ with 
Table 3

Dynamical Properties of ACT 2008 Clusters

\begin{tabular}{|c|c|c|c|c|c|}
\hline ACT Descriptor & $N_{\text {gal }}{ }^{\mathrm{a}}$ & $z_{\mathrm{BI}}$ & $\begin{array}{c}S_{\mathrm{BI}} \\
\left(\mathrm{km} \mathrm{s}^{-1}\right)\end{array}$ & $\begin{array}{c}r_{200 c} \\
\left(h_{70}^{-1} \mathrm{kpc}\right)\end{array}$ & $\begin{array}{c}M_{200 c} \\
\left(10^{14} h_{70}^{-1} M_{\odot}\right)\end{array}$ \\
\hline ACT-CL J0102-4915 & 89 & $0.8701 \pm 0.0009$ & $1321 \pm 106$ & $1789 \pm 140$ & $16.3 \pm 3.8$ \\
\hline ACT-CL J0215-5212 & 55 & $0.4801 \pm 0.0009$ & $1025 \pm 102$ & $1736 \pm 173$ & $9.6 \pm 2.8$ \\
\hline ACT-CL J0232-5257 & 64 & $0.5559 \pm 0.0007$ & $884 \pm 110$ & $1438 \pm 177$ & $5.9 \pm 2.2$ \\
\hline ACT-CL J0235-5121 & 82 & $0.2777 \pm 0.0005$ & $1063 \pm 101$ & $2007 \pm 190$ & $11.9 \pm 3.4$ \\
\hline ACT-CL J0237-4939 & 65 & $0.3344 \pm 0.0007$ & $1280 \pm 89$ & $2339 \pm 162$ & $20.0 \pm 4.2$ \\
\hline ACT-CL J0304-4921 & 71 & $0.3922 \pm 0.0007$ & $1109 \pm 89$ & $1971 \pm 155$ & $12.7 \pm 3.0$ \\
\hline ACT-CL J0330-5227 & 71 & $0.4417 \pm 0.0008$ & $1238 \pm 98$ & $2138 \pm 166$ & $17.1 \pm 4.0$ \\
\hline ACT-CL J0346-5438 & 88 & $0.5297 \pm 0.0007$ & $1075 \pm 74$ & $1770 \pm 122$ & $10.7 \pm 2.2$ \\
\hline ACT-CL J0438-5419d & 65 & $0.4214 \pm 0.0009$ & $1324 \pm 105$ & $2310 \pm 182$ & $21.1 \pm 5.0$ \\
\hline ACT-CL J0509-5341 ${ }^{\mathrm{e}}$ & 76 & $0.4607 \pm 0.0005$ & $846 \pm 111$ & $1451 \pm 189$ & $5.5 \pm 2.1$ \\
\hline ACT-CL J0521-5104 ${ }^{\mathrm{f}}$ & 24 & $0.6755 \pm 0.0016$ & $1150 \pm 163$ & $1744 \pm 245$ & $12.1 \pm 5.1$ \\
\hline ACT-CL J0528-5259 & 55 & $0.7678 \pm 0.0007$ & $928 \pm 111$ & $1337 \pm 159$ & $6.1 \pm 2.2$ \\
\hline ACT-CL J0546-5345 & 48 & $1.0663 \pm 0.0014$ & $1082 \pm 187$ & $1319 \pm 226$ & $8.1 \pm 4.2$ \\
\hline ACT-CL J0559-5249i & 31 & $0.6091 \pm 0.0014$ & $1219 \pm 118$ & $1916 \pm 184$ & $14.9 \pm 4.3$ \\
\hline ACT-CL J0616-5227 & 18 & $0.6838 \pm 0.0019$ & $1124 \pm 165$ & $1699 \pm 244$ & $11.2 \pm 4.9$ \\
\hline ACT-CL J0707-5522 & 58 & $0.2962 \pm 0.0005$ & $832 \pm 82$ & $1561 \pm 156$ & $5.7 \pm 1.7$ \\
\hline
\end{tabular}

Notes.

a Number of spectroscopically confirmed members, after applying the selection procedure of Section 3.2.

b "El Gordo" (Menanteau et al. 2012) and SPT-CL J0102-4915 (Williamson et al. 2011).

c A3128 (NE) (Werner et al. 2007).

${ }^{\mathrm{d}}$ PLCK G262.7-40.9 (Planck Collaboration 2011a) and SPT-CL J0438-5419 (Williamson et al. 2011).

e SPT-CL J0509-5341 (Staniszewski et al. 2009).

${ }^{\mathrm{f}}$ SCSO J052113-510418 (Menanteau et al. 2010b) and SPT-CL J0521-5104 (Vanderlinde et al. 2010).

g SPT-CL J0528-5259 (Staniszewski et al. 2009) and SCSO J052803-525945 (Menanteau et al. 2010b).

h SPT-CL J0547-5345 (Staniszewski et al. 2009).

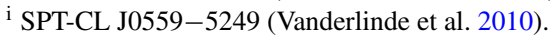

individual errors as measured in this work (i.e., $\lesssim 100 \mathrm{~km} \mathrm{~s}^{-1}$ ), this correction is $<0.1 \%$ (and even lower for more massive clusters), and it is therefore not considered here. ${ }^{23}$

\section{DYNAMICAL MASSES}

In this section, we use the velocity dispersions measured in the previous section to estimate cluster masses. The dynamical state of each cluster is also studied, including signs of substructure and the fraction and influence of emission-line galaxies in the cluster population. Both factors can, in principle, bias the velocity dispersion and thus the dynamical mass of the cluster. Moreover, they are not expected to be completely independent, since emission-line galaxies are generally newly incorporated galaxies, which might mean recent (or near-future) mergers involving the main cluster (Moore et al. 1999; Book \& Benson 2010).

\subsection{Dynamical Mass Estimates}

The relationship between velocity dispersions and masses has been the focus of several studies. As a first-order approach, Heisler et al. (1985) studied simple variations of the Virial Theorem and found that they all behave similarly, and that it is not possible to distinguish among them. Carlberg et al. (1997) compared masses obtained from the Virial Theorem to those obtained with the Jeans equation in observed clusters. They found that the former are biased high by a factor of 10\%-20\% and associated this bias with a surface pressure correction factor of the same order.

\footnotetext{
23 As mentioned before, the errors calculated by RVSAO are smaller than the true cross-correlation errors. Even so, the Danese et al. (1980) correction would be $\ll 1 \%$, and still negligible over the statistical uncertainty in the velocity dispersion.
}

More recently and based on large cosmological simulations, Evrard et al. (2008) concluded that massive $\left(M_{200 c}>10^{14} M_{\odot}\right)$ clusters are, on average, consistent with a virialized state, and find a best-fit scaling relation for dark matter halos described by NFW profiles in a variety of cosmologies. Accordingly, the mass enclosed within $r_{200 c}$ is

$$
M_{200 c}=\frac{10^{15}}{0.7 h_{70}(z)}\left(\frac{\sigma_{\mathrm{DM}}}{\sigma_{15}}\right)^{1 / \alpha} M_{\odot},
$$

where $\sigma_{15}=1082.9 \pm 4.0 \mathrm{~km} \mathrm{~s}^{-1}, \alpha=0.3361 \pm 0.0026$, $h_{70}(z)=h_{70} \sqrt{\Omega_{\Lambda}+(1+z)^{3} \Omega_{M}}$ for a flat cosmology, and $\sigma_{\mathrm{DM}}$ is the $1 \mathrm{D}$ velocity dispersion of the dark matter particles within $r_{200 c}$, which is related to the velocity dispersion of galaxies by a so-called bias factor $b_{v}=S_{\mathrm{BI}} / \sigma_{\mathrm{DM}}$. As summarized by Evrard et al. (2008), the bias factor as currently estimated is $\left\langle b_{v}\right\rangle=1.00 \pm 0.05$. For consistency with previous studies (e.g., Brodwin et al. 2010), we adopt a value $b_{v}=1$, meaning that galaxies are unbiased tracers of the mass in a cluster.

The mass values drawn from Equation (1) are shown in Table 3, and the given errors include uncertainties on the cluster redshift, the velocity dispersion, $\alpha$, and $\sigma_{15}$. The overall uncertainty in the mass is dominated by statistical errors which, in turn, are dominated by the error in the velocity dispersion. The systematics introduced by Equation (1) contribute $<10 \%$ of the uncertainties listed in Table 3. The mass from Equation (1) yields a lower value than the virial mass estimator, as Carlberg et al. (1997) also anticipated.

As indicated by Evrard et al. (2008), Equation (1) holds for primary halos, i.e., clusters where a "main system" can be easily identified and substructure is only marginal. As noted in Section 4.2, a high fraction of the clusters have significant substructure, but none of them show a clear bimodal distribution 
Table 4

Substructure in ACT 2008 Clusters

\begin{tabular}{|c|c|c|c|c|c|c|c|c|}
\hline Cluster & $z$ & $\begin{array}{c}\left|v_{\text {pec }}\right|^{\mathrm{a}} \\
\left(\mathrm{km} \mathrm{s}^{-1}\right)\end{array}$ & $\left|v_{\mathrm{pec}}\right| / S_{\mathrm{BI}}$ & $\begin{array}{c}\Delta r^{\mathrm{b}} \\
(\operatorname{arcsec})\end{array}$ & $\Delta r / r_{200 c}$ & s.1. $(\mathrm{DS})^{\mathrm{c}}$ & \multicolumn{2}{|c|}{ Disturbed $^{\mathrm{d}}$} \\
\hline ACT-CL J0102-4915 e & 0.870 & $10 \pm 169$ & $0.01 \pm 0.13$ & 68 & 0.30 & $0.48_{-0.02}^{+0.13}$ & 010 & $\overline{\text { Yes }}$ \\
\hline ACT-CL J0215-5212 & 0.480 & $1171 \pm 153$ & $1.14 \pm 0.19$ & 33 & 0.12 & $0.02_{-0.01}^{+0.00}$ & 101 & Yes \\
\hline ACT-CL J0232-5257 & 0.556 & $37 \pm 129$ & $0.04 \pm 0.14$ & 35 & 0.15 & $0.11_{-0.05}^{+0.11}$ & 000 & No \\
\hline ACT-CL J0235-5121 & 0.278 & $138 \pm 137$ & $0.13 \pm 0.13$ & 44 & 0.09 & $0.04_{-0.03}^{+0.01}$ & 001 & Yes \\
\hline ACT-CL J0237-4939 & 0.334 & $261 \pm 174$ & $0.20 \pm 0.14$ & 78 & 0.16 & $<0.01$ & 001 & Yes \\
\hline ACT-CL J0304-4921 & 0.392 & $151 \pm 157$ & $0.14 \pm 0.14$ & 22 & 0.06 & $0.04_{-0.03}^{+0.09}$ & 001 & No \\
\hline ACT-CL J0330-5227 & 0.442 & $424 \pm 167$ & $0.34 \pm 0.14$ & 44 & 0.12 & $0.21_{-0.02}^{+0.27}$ & 100 & No \\
\hline ACT-CL J0346-5438 & 0.530 & $263 \pm 125$ & $0.24 \pm 0.12$ & 16 & 0.06 & $0.23_{-0.07}^{+0.05}$ & 100 & No \\
\hline ACT-CL J0438-5419 & 0.421 & $392 \pm 172$ & $0.30 \pm 0.13$ & 10 & 0.02 & $0.03_{-0.02}^{+0.01}$ & 101 & Yes \\
\hline ACT-CL J0509-5341 & 0.461 & $361 \pm 134$ & $0.42 \pm 0.17$ & 114 & 0.46 & $0.08_{-0.03}^{+0.04}$ & 110 & Yes \\
\hline ACT-CL J0521-5104 f & 0.676 & $440 \pm 292$ & $0.37 \pm 0.25$ & 37 & 0.15 & $\cdots$ & $00-$ & No? \\
\hline ACT-CL J0528-5259 & 0.768 & $144 \pm 177$ & $0.16 \pm 0.19$ & 50 & 0.28 & $0.30_{-0.02}^{+0.07}$ & 010 & No \\
\hline ACT-CL J0546-5345 & 1.066 & $541 \pm 163$ & $0.50 \pm 0.17$ & 20 & 0.13 & $0.02_{-0.02}^{+0.04}$ & 101 & Yes \\
\hline ACT-CL J0559-5249 & 0.609 & $233 \pm 241$ & $0.19 \pm 0.20$ & 9 & 0.03 & $0.13_{-0.06}^{+0.13}$ & 000 & No \\
\hline ACT-CL J0616-5227 f & 0.684 & $685 \pm 268$ & $0.61 \pm 0.25$ & 29 & 0.12 & $\ldots$ & $10-$ & Yes? \\
\hline ACT-CL J0707-5522 & 0.296 & $402 \pm 140$ & $0.48 \pm 0.18$ & 19 & 0.05 & $0.34_{-0.15}^{+0.04}$ & 100 & No \\
\hline
\end{tabular}

Notes. Redshifts are listed for reference.

a Absolute value of the peculiar velocity of the BCG in the cluster rest frame (see Section 4.2.1). The uncertainties consider the error on the BCG redshift as twice that given by RVSAO.

${ }^{\mathrm{b}}$ Offset between the BCG and the SZ peak as found in the $Y_{200 c}$ analysis (see Sections 2.1 and 4.2.2 for details).

${ }^{c}$ Significance level of the DS test. Uncertainties are computed at the 75\% level (see Section 4.2.3 for details).

"Each ordered number represents one of the tests listed in the table: " 1 " means the test shows evidence for substructure and " 0 " means it does not.

e This cluster is classified as "disturbed" based on the results of Menanteau et al. (2012). See the text for details.

f There are too few members observed for the DS test to be reliable. The classification is left as a tentative one, and these clusters are excluded from the analysis of Section 6.1 (see Section 4.2.4).

in velocity, and we therefore assume that Equation (1) is applicable to all the clusters in the sample.

The radius $r_{200 c}$ is also listed for each cluster in Table 3. These have been calculated using $M_{200 c}$ and assuming spherical clusters (i.e., $M_{200 c}=200 \rho_{c} \times 4 \pi r_{200 c}^{3} / 3$ ).

\subsection{Substructure}

It is becoming widely accepted that substructure is a common feature of galaxy clusters, and that its presence (or lack thereof) is related to the degree of relaxation and hence the validity of the hydrostatic equilibrium hypothesis (e.g., Battaglia et al. 2012, and references therein). While X-ray observations can reveal the presence of substructure in the plane of the sky, velocity information can reveal substructure in the radial direction. From $\mathrm{X}$-ray observations over a wide range in masses at $z \lesssim 0.3$, Schuecker et al. (2001) find that $(52 \pm 7) \%$ of galaxy clusters present significant substructure. Girardi et al. (1997) find that out of 44 optically selected local $(z \leqslant 0.15)$ clusters, $15(38 \%)$ show significant signs of substructure based on their dynamics. Girardi et al. (1997) argue, on the other hand, that substructure found in clusters that show a unimodal velocity distribution (i.e., where the substructure is not of comparable size to the cluster itself) does not influence the velocity dispersion (hence mass) measurements.

In general, a non-negligible fraction of the galaxy clusters in a sample will have biased mass measurements due to substructure. These results highlight the need for a correct estimation of the degree to which galaxy clusters seem to be relaxed or in the process of merging.
One very basic test for substructure involves the distribution of measured velocities. In fact, however, none of our velocity histograms show clear evidence for a bi- or multi-modal distribution, and the velocity dispersions $S_{\mathrm{BI}}$ are consistent with Gaussian velocity dispersions (i.e., with the standard deviation), in all cases, within $1 \sigma$. So, in the following, we employ three specific tests that take advantage of the three-dimensional (3D) information provided by the optical spectroscopy to assess the dynamical state of the clusters from a wide perspective. Table 4 summarizes the substructure analysis.

\subsubsection{D: BCG Peculiar Velocity}

For a cluster that is relaxed, the peculiar velocity of the BCG should be close to zero (Quintana \& Lawrie 1982; Oegerle \& Hill 2001; but see Pimbblet et al. (2006) for a likely counterexample). Oegerle \& Hill (2001) find that the dispersion of BCG peculiar velocities is $\sim 160 \mathrm{~km} \mathrm{~s}^{-1}$ for a median $S_{\mathrm{BI}} \sim 800 \mathrm{~km} \mathrm{~s}^{-1}$. Using a sample of 452 Abell clusters, Coziol et al. (2009) find that BCGs have a median peculiar velocity $0.32 S_{\mathrm{BI}}$ and that $41 \%$ of BCGs have velocities different from zero at the $2 \sigma$ level, but note that this number is comparable to the fraction of clusters that show signs of substructure. In summary, velocities consistent with zero are not necessarily expected. Dominant (D/cD) BCGs, however, are mostly found in the low peculiar velocity regime. Thus, here clusters are (provisionally) considered as disturbed if their BCG has a peculiar velocity different from zero at the $2 \sigma$ level where, following Coziol et al. (2009), the fractional 
uncertainties are given by

$$
\Delta\left(v_{\mathrm{pec}} / S_{\mathrm{BI}}\right)=\frac{1}{S_{\mathrm{BI}}} \sqrt{\left(\Delta v_{\mathrm{pec}}\right)^{2}+\left(\frac{v_{\mathrm{pec}} \Delta S_{\mathrm{BI}}}{S_{\mathrm{BI}}}\right)^{2}},
$$

where $\Delta v_{\text {pec }}=\sqrt{S_{\mathrm{BI}}^{2} / N_{\text {gal }}+\left(\Delta v_{\mathrm{BCG}}\right)^{2}}$ is the error in the peculiar velocity, and $\Delta v_{\mathrm{BCG}}$ is twice the cross-correlation error estimated by RVSAO, which is a conservative correction (Quintana et al. 2000).

Eight clusters meet this criterion, which will be coupled with similarly chosen criteria in the 2D and 3D analyses before selecting which clusters have significant evidence for substructure.

\subsubsection{D: Projected BCG-SZE Offset}

Under the hypothesis of hydrostatic equilibrium, galaxies closely trace the total mass distribution in the cluster and thus the BCG is located at the peak of the gravitational potential. If the cluster is virialized, the gas should also follow the mass distribution. Deviation from this scenario may be quantified by an offset between the BCG (i.e., dark matter) and the SZE (i.e., gas) peak. This, of course, is sensitive to offsets projected in the sky, unlike the preceding and following tests.

ACT has a beam of 1:4 (FWHM) at $148 \mathrm{GHz}$ (Hincks et al. 2010) and the uncertainties in the determination of the position of each cluster are of order $10^{\prime \prime}-15^{\prime \prime}$. We therefore list the projected offset in arcseconds in Table 4; offsets $\lesssim 15^{\prime \prime}$ are within ACT's positional uncertainty and should therefore not be considered physical offsets. Lin \& Mohr (2004) find that $>80 \%$ of BCGs are offset from the peak gas emission by $\Delta r / r_{200 c}<0.2$. Moreover, Skibba et al. (2011) find that $\sim 40 \%$ of BCGs do not sit at the minimum of the potential well in clusters. Column 6 of Table 4 lists the projected offset between the BCG and the SZE peak for each cluster relative to the characteristic scale of the cluster $r_{200 c}$.

We choose $\Delta r / r_{200 c} \sim 0.20$ as the threshold between (tentatively classified) relaxed and disturbed clusters, based on the results of Lin \& Mohr (2004). In this case, only three clusters-ACT-CL J0102-4915, ACT-CL J0509-5341, and ACT-CL J0528-5259-have values over the threshold. Given that the chance of LOS substructure should be the same as that of substructure in the plane of the sky, ${ }^{24}$ this might be too stringent a limit. Moreover, the findings of Skibba et al. (2011) argue that this might not be a very reliable test for substructure, but we include it for completeness. The three clusters that meet this criterion have offsets on the order of an arcminute, far beyond uncertainties in the ACT SZE centroids, and therefore qualify as physical offsets.

It is worth noting that positions estimated in our analysis differ from those reported in Marriage et al. (2011a), typically by $\approx 20^{\prime \prime}$. There are two exceptions, however: the estimated centers for ACT-CL J0509-5341 and ACT-CL J0707-5522 have changed by $91^{\prime \prime}$ and $119^{\prime \prime}$, respectively. These two clusters are also the clusters with the lowest $\mathrm{S} / \mathrm{N}$, as can be seen from Table 1, so these large shifts are attributed to this fact.

\subsubsection{D: DS Test}

By studying a large sample of statistical tests for substructure in galaxy clusters, Pinkney et al. (1996) have shown that the DS

\footnotetext{
24 In fact, the latter should be approximately twice as large, given the number of dimensions covered by the plane of the sky and the LOS
}

test (Dressler \& Shectman 1988) is the most sensitive test when used individually. The test has the ability not only to detect the presence of substructure but also to locate the latter in projected space (in the ideal cases of substructure not overlapping with the main system either in velocity or in projected space) and is based in the detection of localized subgroups of galaxies that deviate from the global distribution of velocities by use of the parameter $\Delta=\Sigma_{i} \delta_{i}$, where

$$
\delta_{i}^{2}=\frac{N_{\text {local }}}{\sigma^{2}}\left[\left(\bar{v}_{i}-\bar{v}\right)^{2}+\left(\sigma_{i}-\sigma\right)^{2}\right]^{2}
$$

is computed for each cluster member, where $\bar{v}_{i}$ and $\sigma_{i}$ are the mean and standard deviation of the velocity distribution of the $N_{\text {local }}$ members closest to the $i$ th member, and $\bar{v}$ and $\sigma$ are the mean and standard deviation of the velocity distribution of all the cluster members. The significance level (s.l.) of the test is obtained by shuffling the velocities of each galaxy via a bootstrap resampling technique with 5000 iterations. Although the common use is that $N_{\text {local }}=\sqrt{N_{\text {gal }}}$, in this work $\Delta$ is calculated for $N_{\text {local }}$ ranging from 5 to 12 . The uncertainties in the s.l. are given by the second-maximum and second-minimum s.l. for each cluster when varying $N_{\text {local }}$ (i.e., they correspond to $\sim 75 \%$ level uncertainties), and the central value is given by the median. A large uncertainty (i.e., dependence on $N_{\text {local }}$ ) might also be indicative of substructure, but we do not include this in the analysis.

As shown by Pinkney et al. (1996), the false positive rate for the DS test is $<1 \%,<4 \%$, and $9 \%$ for an s.l. of $1 \%, 5 \%$, and $10 \%$, respectively, for member samples as large as ours in clusters simulated by Gaussian distributions of galaxies. The threshold for substructure detection is set therefore at 5\% s.l. within uncertainties; seven clusters meet this criterion. Given a false positive rate of $4 \%$, there is a $25 \%$ chance that a cluster has been spuriously classified as disturbed by the DS test.

\subsubsection{Substructure Results}

Clusters have been identified as merging systems if they meet at least two of the three conditions explained above, or if they have an s.l. of the DS test strictly below 5\% within uncertainties. Although the second of the three conditions depends on the projected spatial distribution, it is clear that this analysis is biased toward LOS substructure.

ACT-CL J0102-4915 ("El Gordo") is a special case, as it does not show evidence for merging from the dynamical information alone. However, both the spatial galaxy distribution and X-ray surface brightness distribution reveal that this is a very complex system where two massive clusters are interacting close to the plane of the sky (Menanteau et al. 2012).

On the other hand, ACT-CL J0616-5227 is tentatively considered as a merging cluster given the high peculiar velocity of the BCG, but the DS test was not performed for this cluster given the low number of members. The latter note also applies to ACT-CL J0521-5104, although this cluster is tentatively considered relaxed. These two clusters have been excluded from the analysis of Section 6.1.

The last column of Table 4 states whether a cluster is considered to be relaxed ("No") or disturbed ("Yes"), while the previous column lists whether each cluster shows ("1") or does not show ("0") signs of substructure in each of the tests, as defined above. Combining the criteria used, 7 out of 14 clusters show signs of merger activity (or 8 of 16, if we include ACT-CL J0521-5104 and ACT-CL J0616-5227). This number is consistent with previous optical and X-ray studies of local 


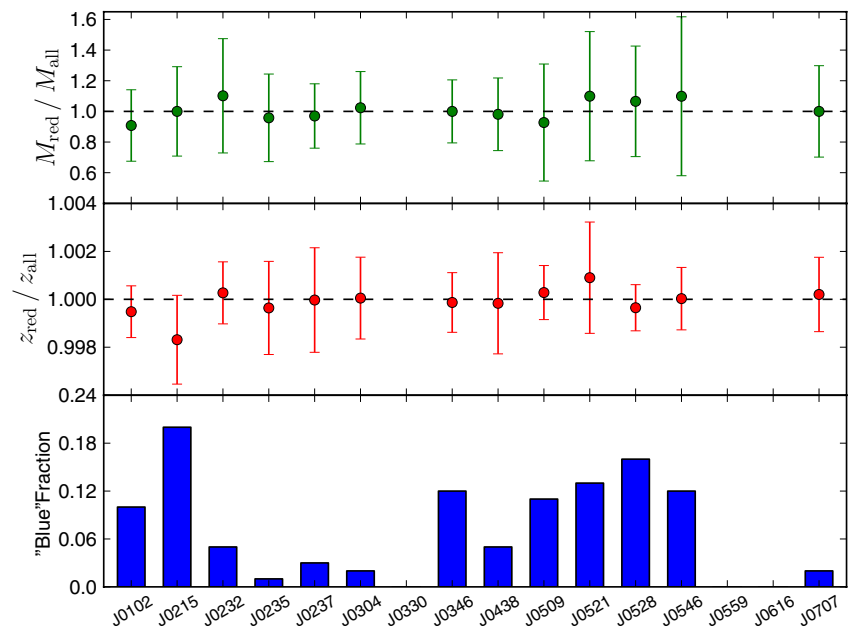

Figure 3. Top two panels show, for each cluster indicated on the horizontal axis, the ratios of dynamical masses (top) and cluster redshifts (middle) when only the absorption-line ("red") galaxies or all galaxies are used for the analysis. Error bars are given by $\Delta M_{\text {all }} / M_{\text {all }}$ and $\Delta z_{\text {all }} / z_{\text {all }}$, respectively. The dashed line in each panel marks a ratio of unity. The bottom panel shows the observed fraction of galaxies with emission lines ("blue"). Cluster names have been shortened for clarity; data points in the top and middle panels have been omitted for the three clusters with blue fractions equal to 0 .

(A color version of this figure is available in the online journal.)

clusters (e.g., Girardi et al. 1997; Schuecker et al. 2001) and is also consistent with the X-ray follow-up of SPT SZE-detected clusters by Andersson et al. (2011). They find that 9 out of 15 SZE-selected clusters show signs of substructure based purely on X-ray morphology.

\subsection{The Influence of Emission-line Galaxies}

Clusters of galaxies are mostly populated by passive galaxies. Late-type galaxies are preferentially found in the outskirts of clusters and associated with infalling groups. They therefore tend to show a different velocity distribution (Biviano \& Katgert 2004). Girardi et al. (1996) find that 29\% (53\%) out of a sample of 17 nearby clusters show differences in the velocity dispersion and $24 \%(47 \%)$ in the mean velocity, at the $2 \sigma(1 \sigma)$ level. Simulations also show that, where blue galaxies are found (i.e., outside the core), they tend to have a higher velocity dispersion than red galaxies (Springel et al. 2001b). The way blue galaxies are distributed in the cluster (both in space and in velocity) depends, however, on the history of each cluster (Biviano \& Katgert 2004). The issue is complex; for example, Aguerri \& Sánchez-Janssen (2010) find no difference in the fraction of blue galaxies between relaxed and disturbed clusters.

Although our target selection procedure should not be strongly biased against emission-line galaxies, the observations have not been designed to study this effect and the spectroscopic samples have emission-line fractions of $\lesssim 10 \%$ in most cases. This number does not necessarily reflect the actual fraction in the clusters and could be taken as a lower limit for it. In spite of all this, we briefly study the effect that blue ${ }^{25}$ galaxies might have on the results.

Figure 3 shows, in the top and middle panels, respectively, the variation of the mass measurement and the cluster redshift when

25 Although the classification is done purely based on the spectral features of each galaxy (with or without emission lines), we sometimes speak of blue and red, instead of emission- and absorption-line galaxies, respectively, for convenience. blue (i.e., emission line) galaxies are, and are not, included. The null hypothesis (i.e., no bias) corresponds to $M_{\text {red }} / M_{\text {all }}=1$. Uncertainties in $z_{\text {red }} / z_{\text {all }}$ and $M_{\text {red }} / M_{\text {all }}$ are given by $\Delta z_{\text {all }} / z_{\text {all }}$ and $\Delta M_{\text {all }} / M_{\text {all }}$, respectively, where $\Delta z_{\text {all }}$ and $\Delta M_{\text {all }}$ are the uncertainties reported in Table 3. Within uncertainties, neither cluster redshifts nor dynamical masses change when including, or not, emission-line galaxies. Data points are not shown for the three clusters with fractions of emission-line galaxies-which are shown in the bottom panel of Figure 3-equal to zero. These three clusters have, by definition, $M_{\text {red }} / M_{\text {all }}=z_{\text {red }} / z_{\text {all }}=1$. Note that for the red-only analysis, the blue galaxies are removed before the selection process (i.e., $N_{\text {red }}$ does not necessarily equal $\left.N_{\text {gal }}-N_{\text {blue }}\right)$.

The redshifts, velocity dispersions, and corresponding masses in Table 3 have been calculated using all galaxies, since blue galaxies do not bias our mass (or redshift) measurements. This is, in turn, consistent with the findings of Aguerri \& SánchezJanssen (2010).

\section{SZE-MASS SCALING RELATIONS}

Both Vanderlinde et al. (2010) and Sehgal et al. (2011) have shown that, given an accurate calibration of the SZE-mass scaling relation, the inclusion of the ACT or SPT cluster samples can lead to significant improvements in cosmological parameter uncertainties, particularly $w$ and $\sigma_{8}$, over WMAP-7 only constraints. These results have recently been confirmed by Benson et al. (2013) using X-ray observations. However, without a precise SZE-mass scaling relation, these cluster samples do not provide significant improvements in constraining cosmological parameters.

Observations have shown that the SZE signal and mass of a cluster can be related by a power law (Benson et al. 2004; Bonamente et al. 2008; Melin et al. 2011). While most simulations seem to confirm this (da Silva et al. 2004; Motl et al. 2005; Nagai 2006), others suggest that certain effects (e.g., AGN feedback) can cause deviations from a single powerlaw dependence (Battaglia et al. 2012). In this work, we restrict ourselves to a power-law relation between dynamical mass (see Section 4 and Table 3) and each SZE estimator measured from the ACT data (see Section 2.1.2 and Table 1) of this form:

$$
\begin{gathered}
\frac{M_{200 c}}{h_{70}^{-1} M_{\odot}}=10^{A}\left(\frac{\tilde{y_{0}} E(z)^{-2}}{5 \times 10^{-5}}\right)^{B} \\
\frac{M_{200 c}}{h_{70}^{-1} M_{\odot}}=10^{A}\left(\frac{y_{0} E(z)^{-2}}{7 \times 10^{-5}}\right)^{B} \\
\frac{M_{200 c}}{h_{70}^{-1} M_{\odot}}=10^{A}\left(\frac{Y_{200 c} D_{A}(z)^{2} E(z)^{-2 / 3}}{5 \times 10^{-5} h_{70}^{-2} \mathrm{Mpc}^{2}}\right)^{B} .
\end{gathered}
$$

Here, $D_{A}(z)$ is the angular diameter distance in Mpc, $M_{200 c}$ is in units of $h_{70}^{-1} M_{\odot}$, and $E(z)=\left[\Omega_{M}(1+z)^{3}+\Omega_{\Lambda}\right]^{1 / 2}$. We refer to $B$ as the (logarithmic) slope of the scaling relations. The self-similar predictions are 1 and 0.6 for the $y_{0}$ and $Y_{200 c}$ scaling relations, respectively (e.g., Bonamente et al. 2008; Marriage et al. 2011a). Equations (4) are convenient forms of parameterizing the scaling relations if one wants to predict the mass of a cluster using SZE observations. 


\subsection{Selection Biases}

Before proceeding, we consider the selection biases that can affect our study when fitting the scaling relations (see Mantz et al. 2010a, for a pedagogical description).

The first one is Eddington (1913) bias, which results from the asymmetry of the steep underlying mass function (e.g., Jenkins et al. 2001; Tinker et al. 2008), coupled with measurement errors, which introduces a net shift in mass due to the statistical fluctuations of the measurement of the mass proxy in the mass-observable (in this case, SZE signal) relation. While there are analytical prescriptions to account for Eddington bias (e.g., Mortonson et al. 2011), we have assessed the effect of measurement errors in our sample by simulating measurement uncertainties, comparable to those of our data, in the simulations of Sehgal et al. (2010) and re-calculating the scaling relation for 10,000 realizations. We find that the scaling relations in these simulations are unchanged when introducing measurement uncertainties. We thus estimate that Eddington bias can be safely neglected in this case.

The second bias is produced by the intrinsic scatter in the observable: clusters with mean SZE fluxes at the detection limit whose signals scatter up will make it into the sample, while those that scatter down will not. We refer to this effect as a flux bias. ${ }^{26}$ We use the simulations of Sehgal et al. (2010) including prescriptions for both AGN and supernova feedback, plus a realistic modeling of non-thermal pressure support (Bode et al. 2012), to investigate this effect in our sample. We measure $\widetilde{y_{0}}$ from the simulated clusters as in Section 2.1.2 and subsequently apply a cut $\tilde{y}_{0} T_{\mathrm{CMB}}>150 \mu \mathrm{K}$ to the simulated data. This "observational" cut approximately reflects the detection threshold of the observed cluster sample. This procedure mimics the observational situation with the exception that it assumes a constant noise level throughout the survey. The ACT sample is defined in terms of an $\mathrm{S} / \mathrm{N}$ limit, although the noise level is approximately constant except near the edges of the map (Marriage et al. 2011a, 2011b). Within our sample, there is potentially only one cluster (ACTCL J0707-5522) whose flux bias correction is not accurately described by this procedure because it sits in a high-noise region in the maps. If this cluster is removed from the sample, the change in the corrected scaling laws is negligible.

In practice, clusters within a mass range from $M$ to $M+$ $\Delta M$ (where $\Delta M=10^{14} h_{70}^{-1} M_{\odot}$ ) are extracted from the simulations and the average $\widetilde{y_{0}}$ value of the extracted subsample is determined both with and without a detection threshold. The ratio of $\widetilde{y_{0}}$ values represents a statistical estimate of the flux bias factor for clusters within this mass range. At the low-mass end of the cluster sample, the bias correction factor is $\sim 0.8$, while for clusters with $M_{200 c}>9 \times 10^{14} M_{\odot}$ the correction factor is close to unity. A continuous smooth curve is fitted to the bias correction factors as a function of mass and applied individually to each cluster's SZE measurements. The uncertainty on the mass is propagated through the bias correction factors and then into the corrected measurements. To test the dependence of the correction on the adopted cluster physics, the procedure is repeated both for a model with no star formation or AGN feedback and only thermal pressure support (an "adiabatic" model) and for a model with a generous $20 \%$

\footnotetext{
26 This effect has often been called "Malmquist bias." However, the term "Malmquist bias" was coined related to the specific problem of an error in average distance modulus measurements tied with a magnitude-limited sample and so is inappropriate here; see the review by Teerikorpi (1997).
}

Table 5

Best-fit Parameters of Scaling Relations

\begin{tabular}{lcccc}
\hline \hline Relation & $B_{\mathrm{SS}}{ }^{\mathrm{a}}$ & $A$ & $B$ & $\sigma_{M Y}$ \\
\hline$\widetilde{y_{0}}$ corr $-M_{200 c}$ & $\ldots$ & $15.02 \pm 0.07$ & $0.79 \pm 0.15$ & $0.13 \pm 0.05$ \\
$y_{0}^{\text {corr }}-M_{200 c}$ & 1 & $15.02 \pm 0.07$ & $0.84 \pm 0.14$ & $0.13 \pm 0.05$ \\
$Y_{200 c}^{\text {corr }}-M_{200 c}$ & 0.6 & $14.99 \pm 0.07$ & $0.48 \pm 0.11$ & $0.18 \pm 0.10$ \\
\hline
\end{tabular}

Notes. Uncertainties have been estimated through bootstrap resampling.

a Expected logarithmic slope from self-similar evolution.

non-thermal pressure support, constant with mass, radius, and redshift (Bode et al. 2009) as limiting cases, again accounting for the uncertainty in the mass measurements. The variation in the scaling relations given by these simulations is well within reported errors on the scaling relations, both in the normalizations and in the slopes.

We apply the same bias correction factors to each of the different SZE estimators. This is a reasonable approach since the latter are all based on matched filters with kernels of similar scales. To distinguish the bias-corrected values hereafter, we label them with a superscript "corr."

\subsection{Best-fit Scaling Relations}

We use the Bivariate Correlated Errors and intrinsic Scatter (BCES) $X_{2} \mid X_{1}$ algorithm for linear regression (Akritas \& Bershady 1996), which takes into account correlated measurement errors in both variables and intrinsic scatter, to find the best-fit slopes and normalizations of the power-law scaling relations given by Equations (4). The results are shown in Figure 4, where the solid lines represent the best-fit power laws and the shaded regions are the $1 \sigma$ uncertainties. Table 5 lists the best-fit parameters, where the last column lists the lognormal intrinsic scatter orthogonal to the best-fit line, as described by Pratt et al. (2009). All uncertainties have been estimated through bootstrap resampling. Different symbols identify the dynamical state of each cluster (see Section 6.1). It is important to mention that, in the case of the $Y_{200 c}-M_{200 c}$ scaling relation, error correlations are taken into account in the fitting (see also Section 6.4).

All three SZE estimators correlate well with dynamical mass, with Pearson's $r$-values of $0.78,0.82$, and 0.86 for ${\widetilde{y_{0}}}^{\text {corr }}, y_{0}^{\text {corr }}$, and $Y_{200 c}^{\text {corr }}$, respectively. The fractional errors on the slopes are similar, ranging from $16 \%$ for $\tilde{y}_{0}{ }^{\text {corr }}-M_{200 c}$ to $20 \%$ for $Y_{200 c}^{\text {corr }}-M_{200 c}$, while $\sigma_{M Y}$ is roughly the same for all three SZE estimators (but see Section 6.4). These values are consistent with those found in simulations, which have some dependence on the input cluster physics and are of order 10\%-15\% for largeaperture integrations, such as $Y_{200 c}$ (Nagai 2006; Yang et al. 2010; Battaglia et al. 2012). We find that the intrinsic scatter of the scaling relations for the central measures is low and similar to that of $Y_{200 c}^{\text {corr }}-M_{200 c}$, although numerical simulations predict a higher dependence on gas physics and projection effects for central estimates (e.g., Motl et al. 2005; Shaw et al. 2008).

As a consistency check, the best-fit power laws have also been estimated using the publicly available code by Kelly (2007), which also takes into account measurement errors in both variables and intrinsic scatter. The scaling relations estimated using this method yield results that are consistent with those listed in Tables 5 and 6, both in magnitude and uncertainties, for $A, B$, and $\sigma_{M Y}$. 

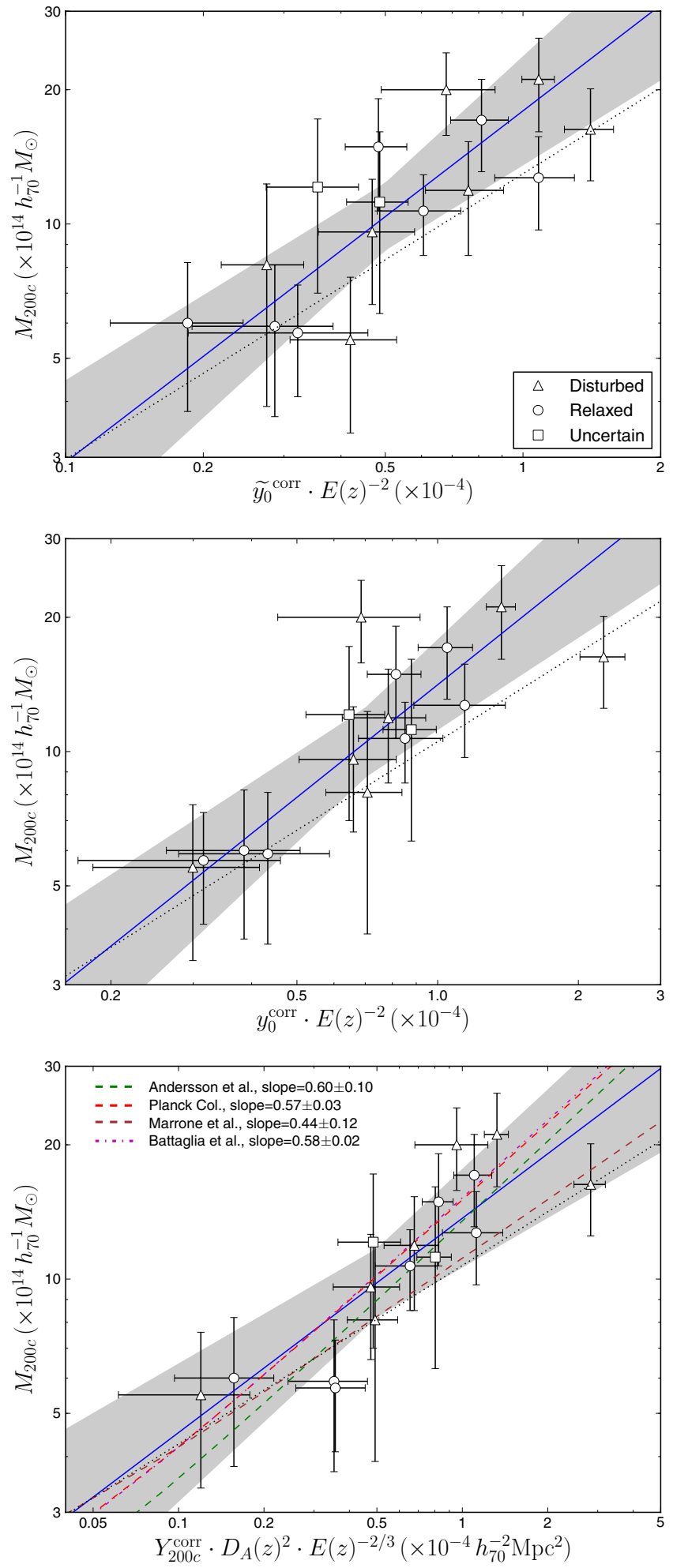

Figure 4. Scaling relations between SZE estimators and dynamical mass for the match-filtered amplitude $\tilde{y_{0}}$ (top), the central Compton parameter $y_{0}$ (middle), and $Y_{200 c}$, the Compton $y$-parameter integrated out to $r_{200 c}$ (bottom), all including three-season ACT data. All estimators have been scaled as indicated in the axis labels (see Equations (4)) and data points have been corrected for flux bias as detailed in the text. Solid blue lines show the best-fit power laws, with the $1 \sigma$ uncertainties marked by the shaded regions (see Table 5). Different symbols identify whether each cluster is disturbed (triangles), relaxed (circles), or not classified (squares). The black dotted line shows the scaling relation found when applying the NFW profile correction described in Section 6.2. Previous estimates of the $Y_{200 c}-M_{200 c}$ scaling relation are shown in the bottom panel with dashed and dot-dashed lines (see the text for details).

(A color version of this figure is available in the online journal.)
Table 6

Best-fit Parameters of Scaling Relations for Selected Subsamples

\begin{tabular}{lcccc}
\hline \hline Relation & Sample & $A$ & $B$ & $\sigma_{M Y}$ \\
\hline$\tilde{y}_{0}{ }^{\text {corr }}-M_{200 c}$ & Disturbed & $14.99 \pm 0.13$ & $0.86 \pm 0.36$ & $0.15 \pm 0.09$ \\
& Relaxed & $15.01 \pm 0.12$ & $0.77 \pm 0.28$ & $0.13 \pm 0.09$ \\
$y_{0}^{\text {corr }}-M_{200 c}$ & Disturbed & $15.03 \pm 0.33$ & $0.78 \pm 0.48$ & $0.18 \pm 0.10$ \\
& Relaxed & $15.01 \pm 0.11$ & $0.93 \pm 0.20$ & $0.09 \pm 0.08$ \\
$Y_{200 c}^{\text {corr }}-M_{200 c}$ & Disturbed & $15.02 \pm 0.13$ & $0.43 \pm 0.17$ & $0.23 \pm 0.18$ \\
& Relaxed & $14.96 \pm 0.11$ & $0.58 \pm 0.19$ & $0.15 \pm 0.15$ \\
\hline
\end{tabular}

Notes. There are seven disturbed and seven relaxed clusters. The scaling relations are in the same form as in Equations (4). Uncertainties have been estimated through bootstrap resampling.

\subsection{Previous Results}

Rines et al. (2010) were the first to present a comparison of SZE fluxes and masses derived from dynamical information, but their sample selection did not allow for the estimation of a scaling relation. Here, we review some SZE-mass scaling relations derived from other observations or mass proxies. While we note that many authors have presented scaling relations in different forms and using a variety of mass proxies, here we compare to those that have done so in the same form as is done here (i.e., correcting by intrinsic evolution in the form of Equations (4)).

When comparing to them, we have converted to values calculated within $r_{200 c}$ by assuming that the mass scales as $M \propto Y^{\gamma}$, where $\gamma$ is the best-fit slope found in each study. Specifically, the conversion from a radius $r_{\Delta}$ to $r_{200 c}$ is done by noting that, if $M_{\Delta}=\alpha M_{200 c}$ (given by an NFW profile) and $Y_{\Delta}=\beta Y_{200 c}$ (given by the A10 profile), then if the scaling relation is of the form $M_{\Delta} \propto Y_{\Delta}^{\gamma}$, it is straightforward that $\left(\alpha M_{200 c}\right) \propto\left(\beta Y_{200 c}\right)^{\gamma}$.

The bottom panel of Figure 4 shows these scaling relations; those where masses were estimated from X-ray observations (Andersson et al. 2011; Planck Collaboration 2011b) and weaklensing measurements (Marrone et al. 2012) are shown with dashed lines, and the dash-dotted line shows the results from hydrodynamical simulations by Battaglia et al. (2012) which include AGN feedback. (The quoted values for the latter are the results at $z=0.5$, which also corresponds to the characteristic redshift of our sample.) The latter authors measure $Y_{200 c}^{\mathrm{cyl}}$, the integrated Compton parameter within a cylinder of radius $r_{200 c}$, which is converted to a spherical measure following Arnaud et al. (2010).

The $Y_{200 c}^{\text {corr }}-M_{200 c}$ scaling relation derived in this work is in good general agreement with the scaling relations cited above, although it is slightly shallower than those derived by Andersson et al. (2011) and Planck Collaboration (2011b) and that predicted by Battaglia et al. (2012), although the large uncertainties prevent any further analysis. Larger samples of clusters should help decrease these error bars.

\section{POSSIBLE BIASES AND SYSTEMATIC EFFECTS}

In this section we explore some effects, both physical and from the analysis, that could be biasing the results of Section 5 . Given the current data set, however, they are all hard to assess, so we have relied on simulations for some of them. A more detailed treatment of these effects will be performed in the future, with a larger sample of clusters. 


\subsection{Scaling Relations for Relaxed and Disturbed Clusters}

Table 6 lists the best-fit scaling relations when separating the sample into relaxed and disturbed clusters according to Table 4 (see Section 4.2). The effect of disturbed clusters, if any, is similar for all SZE estimators and is apparent as a slight, but not significant, change in slope, with disturbed clusters making the slope of the scaling relations $\sim 20 \%$ shallower. While errors on samples of this size are very large, we find that $y_{0}^{\text {corr }}-M_{200 c}$ has the largest decrease in scatter when including only relaxed clusters, and the largest boost for disturbed clusters. However, larger samples of clusters are needed to test whether disturbed systems induce a significant bias, or larger uncertainties, in the scaling relations.

As explained in Section 4.2, neither ACT-CL J0521-5104 nor ACT-CL J0616-5227 has been considered in the present analysis. Including these clusters in either sample does not change the best-fit parameters and only changes the intrinsic scatter by $\lesssim 0.05$, which is within the quoted uncertainties.

\subsection{Systematic Effects from a Reduced Spectroscopic Coverage}

Figure 1 shows that the spectroscopic coverage does not reach $r_{200 c}$ with a significant number of members in many of the clusters studied here. The spectroscopic aperture is defined here as the median BCG-centric distance of the last distanceordered bin of 10 galaxies. This is assumed to be enough so that a measure of the velocity dispersion of these galaxies at such distance is representative of all member galaxies (with and without a redshift measurement) in this bin. The distribution of apertures is asymmetric, with an average $r_{\text {ap }}=0.55_{-0.24}^{+0.36}$ (90th and 10th percentiles), and is a function of the angular diameter distance $D_{A}(z)$ and the size of the cluster (hence the mass). Thus, more massive clusters at lower redshift have the smallest coverage.

Observations and simulations seem to give different answers to what the velocity dispersion profile of a cluster should look like. Simulations show that the velocity dispersion profile for an NFW density profile should be decreasing with radius (e.g., Biviano et al. 2006; Mamon et al. 2010). Most observations, however, find that, on average, the velocity dispersion profile of clusters is flat outside $r \approx 0.5 r_{200 c}$ (e.g., Biviano \& Girardi 2003; Katgert et al. 2004; Faltenbacher \& Diemand 2006; Łokas et al. 2006), although some observations do support the expectations from simulations (e.g., Rines et al. 2003).

Because there are many unknowns in the size of the correction and the cluster properties that drive it, we do not correct our mass measurements by any bias introduced by this reduced coverage. However, we do estimate what the bias could be based on theoretical predictions. We use the velocity dispersion profile predicted by an NFW profile, as derived by Mamon et al. (2010) from $N$-body simulations, using the mass-concentration relation of Duffy et al. (2008). We correct our measurement to a measurement at $r_{200 c}$ using this profile and measure a "corrected" $M_{200 c}$. Since the re-calculation of $r_{200 c}$ for a lower (higher) mass means that we have under(over-)estimated the actual sampling aperture, this procedure is iterated until the radius converges, which takes three to four iterations. The average correction to the velocity dispersion is 0.91 , and the average mass correction derived from Equation (1) is 0.79. The correction to $r_{200 c}$ is of the same order as that of the velocity dispersion, and we use these corrected radii to estimate corrected $Y_{200 c}$, which are on average 0.91 of those reported in Table 1. As mentioned in Section 2.1.2, $\tilde{y}_{0}$ and $y_{0}$ are measured completely independent from the dynamical masses, so these values are not affected. The scaling relations estimated from the corrected numbers are shown in each panel of Figure 4 as a black dotted line. The effect of the correction is to flatten the slopes and lower the normalizations, with $A=\{14.93 \pm 0.06,14.83 \pm 0.06,14.91 \pm 0.06\}$ and $B=\{0.60 \pm 0.09,0.66 \pm 0.08,0.40 \pm 0.09\}$ for $\left\{\tilde{y_{0}}, y_{0}, Y_{200 c}\right\}$. The resulting scatter is slightly lower but consistent with the values reported in Table 5 for all estimators.

We also estimated, for comparison, the correction obtained when applying the surface pressure correction term (The \& White 1986; Girardi et al. 1998), assuming an NFW profile. This correction is directly applied to the mass measurement. The average correction to the present sample is 0.73 . This yields best-fit scaling relations with shallower slopes but consistent with the previous method. However, this correction is applicable specifically to the virial mass measurement (i.e., estimated from the Virial Theorem), so should be taken with care, especially for a population that may be dominated by dynamically disturbed clusters like the present one.

\subsection{The Redshift Evolution of $\tilde{y_{0}}$}

While the functional forms of Equations (4) are well motivated from self-similar evolution for $y_{0}$ and $Y_{200 c}, \widetilde{y_{0}}$ is dependent on the adopted filtering of the maps (Hasselfield et al. 2013) and we have no a priori information on how it should evolve with redshift for a fixed mass. We have explored a range of functional forms for the redshift dependence of Equation (4a) using the set of models discussed in Section 5.1. We find that, while the results are consistent, the models prefer a slightly lower redshift evolution of $\widetilde{y_{0}}$ at fixed mass. Specifically, the scaling resulting from Equation (4a) could, at low $(z \sim 0.3)$ redshifts, bias the masses high (on average) by as much as $\sim 25 \%$. Conversely, at high $(z \sim 1)$ redshifts the masses could be biased low by up to $\sim 35 \%$.

These new mass predictions would, for a variety of redshift parameterizations and for all the clusters in our sample, be within the measured $1 \sigma$ uncertainties. Given the sample size and measurement uncertainties, we have decided to study $\tilde{y}_{0}$ in a similar way to $y_{0}$, to be able to compare more easily the two, which are closely related. As mentioned above, a more detailed study of the functional form of Equations (4) will be performed in future work with a larger sample of clusters.

\section{4. $M_{200 c}-Y_{200 c}$ Correlation}

Since $r_{200 c}$ is derived from dynamical information and used to estimate $Y_{200 c}$, there is non-zero correlation between $Y_{200 c}$ and $M_{200 c}$. As noted in Section 5.2, the best-fit slopes and normalizations listed in Tables 5 and 6 include error correlations between these two parameters. The effect of this correlation is to flatten the relation notably, although within error bars: if not included in the BCES fit, the slope increases to $0.56 \pm 0.11$.

Additionally, as discussed analogously by Becker \& Kravtsov (2011) in the context of the $M_{500 c}-M_{\text {gas }}$ relation, such a correlation will bias the intrinsic scatter measurement low by a factor $\approx 1-\alpha / 3$, where $Y_{200 c} \propto\left(r / r_{200 c}\right)^{\alpha}$ near $r_{200 c}$. By re-calculating $Y_{200 c}$ at different radii around $r_{200 c}$ (see Section 2.1.2), we find $\alpha \simeq 1.18$. The intrinsic scatters of the $Y_{200 c}-M_{200 c}$ relations in Tables 5 and 6 consequently include a correction factor $\approx 1.65$, which makes them larger than the intrinsic scatters for the other relations but consistent within the large error bars. 


\section{DISCUSSION}

\subsection{Individual Clusters}

In this section we list clusters with notable features, including comparison of dynamical masses presented here with previous estimates, where available. ${ }^{27}$ The respective original or alternative names can be found in Table 3 . With respect to notes on optical features of these clusters, the reader is referred to Figures 4-10 of Menanteau et al. (2010a), as appropriate.

\subsubsection{ACT-CL J0102-4915 "El Gordo"}

Located at $z=0.870$, this cluster has the largest SZE signal of all ACT clusters (it is the rightmost data point in all panels of Figure 4) and is one of the most massive clusters of the sample according to its dynamics. This cluster looks elongated in the optical (in fact, it is double-peaked in the galaxy distribution; Menanteau et al. 2012), but there are no clear signs of LOS substructure from the dynamical information. In Menanteau et al. (2012), we show that if the cluster is divided into two subclusters in the process of merging (as suggested by the optical data), they have a mass ratio of order $2: 1$, with a total summed dynamical mass of $M_{200 c}=(24 \pm 7) \times 10^{14} h_{70}^{-1} M_{\odot}$, making this a huge merger between two already massive clusters.

Menanteau et al. (2012) used a multi-wavelength data set combining X-rays, SZE, and the information provided in this work to estimate the cluster mass using several mass proxies which are in statistical agreement, with a combined mass estimate of $M_{200 a}=(21.6 \pm 3.2) \times 10^{14} h_{70}^{-1} M_{\odot}$. The statistical error for the combined mass is likely an underestimate given the complex nature of this massive merging cluster (see Menanteau et al. 2012, for a detailed discussion of the mass measurements in "El Gordo").

\subsubsection{ACT-CL J0215-5212}

As shown in Table 4, this cluster appears to have substructure with a high significance as given by the DS test. More noteworthy, however, is the peculiar velocity of the BCG, $v_{\text {pec }}=1171 \pm 153 \mathrm{~km} \mathrm{~s}^{-1}$, different from zero at $>7.5 \sigma$. This is the only cluster in our sample in which the velocity of the BCG is comparable to the velocity dispersion of the cluster, and the cluster where the emission-line galaxies are most different from the whole population. ACT-CL J0215-5212 has a second galaxy $\sim 23^{\prime \prime}$ away (corresponding to a projected distance of $140 h_{70}^{-1} \mathrm{kpc}$ at $z=0.480$ ) which is only $0.27 \mathrm{mag}$ fainter and has a peculiar velocity of roughly $-660 \mathrm{~km} \mathrm{~s}^{-1}$, and at least three more galaxies within $0.55 \mathrm{mag}$ of the BCG (which is the brightest of all by definition, but is also the one nearest to the optical center of the cluster), all of which have comparable $\left(\sim 1000 \mathrm{~km} \mathrm{~s}^{-1}\right)$ peculiar velocities. On the other hand, this cluster does not significantly depart from any of the scaling relations of Figure 4, showing the complexity of substructure analyses. It also has the highest fraction of emission-line galaxies.

\subsubsection{ACT-CL J0237-4939}

Similar to the previous case, this cluster has three bright galaxies within $2^{\prime}$ of the BCG, which are within 1 mag of the BCG.

\footnotetext{
27 We quote the original mass estimates, given as $M_{500 c}$-also with respect to the critical density of the universe-and assume a typical conversion factor $M_{200 c} \approx 1.6 M_{500 c}$ (Duffy et al. 2008) when comparing with our results. In the particular case of "El Gordo," masses are originally given as $M_{200 a}$, the masses within a radius containing 200 times the average density of the universe. For this cluster, the conversion is $M_{200 c} \approx 0.86 M_{200 a}$.
}

In particular, the second-brightest galaxy is $65^{\prime \prime}\left(310 h_{70}^{-1} \mathrm{kpc}\right)$ away from the BCG, is 0.49 mag fainter, and has a peculiar velocity with respect to the cluster of $\sim 1850 \mathrm{~km} \mathrm{~s}^{-1}$. All this argues in favor of the classification of this cluster as a disturbed system.

\subsubsection{ACT-CL J0330-5227}

As mentioned in Section 2.1.1, this cluster was discovered by Werner et al. (2007) behind A3128 ( $z=0.06)$ using XMM-Newton X-ray observations. The SZE measurement is clearly associated with the background structure while the less massive, foreground cluster has no significant SZE emission (Hincks et al. 2010). The dynamical mass estimated here is significantly higher than that derived by Werner et al. (2007), of $M_{500 c}=3.4 \times 10^{14} h_{70}^{-1} M_{\odot}$. They do caution, however, that their estimate is uncertain, as it is based on an isothermal beta model for the cluster. Being located only $12^{\prime}$ away from A3128 at $z=0.06$ on the sky, this cluster is a clear illustration of the mass selection of SZE surveys, approximately independent of redshift (see Hincks et al. 2010, for further discussion).

\subsubsection{ACT-CL J0438-5419}

ACT-CL J0438-5419 is the only new ACT cluster also reported by the Planck satellite in its early release (PLCKESZ G262.7-40.9; Planck Collaboration 2011a). ${ }^{28}$ It has been followed up with XMM-Newton, with which Planck Collaboration (2011d) estimated a mass $M_{500 c}=(6.9 \pm 0.7) \times 10^{14} h_{70}^{-1} M_{\odot}$ using a $Y_{X}-M$ scaling. This value is $1.6 \sigma$ lower than our dynamical estimate; in fact, this cluster is one of the most massive ones in our sample. However, their reported errors include only statistical effects, so are underestimates of the true errors in the measurement.

This cluster is also reported in Williamson et al. (2011). They estimate a simulation-based SZE-estimated mass $M_{500 c}=$ $(8.2 \pm 2.5) \times 10^{14} h_{70}^{-1} M_{\odot}$, consistent with our dynamical mass estimate.

\subsubsection{ACT-CL J0509-5341}

This was one of the first clusters discovered by SPT (Staniszewski et al. 2009), and the first mass measurements were reported by Menanteau \& Hughes (2009). Vanderlinde et al. (2010) reported a simulation-based SZE estimate of the mass of $M_{500 c}=(4.3 \pm 1.1) \times 10^{14} h_{70}^{-1} M_{\odot}$, and Andersson et al. (2011) estimated an X-ray $Y_{X}$-derived mass from $M_{500 c}=$ $(5.4 \pm 0.6) \times 10^{14} h_{70}^{-1} M_{\odot}$. All previous values are in agreement with our estimate. Consistent with our substructure analysis, Andersson et al. (2011) found that this cluster is a disturbed system based solely on X-ray morphology.

$$
\text { 7.1.7. ACT-CL J0521-5104 }
$$

This cluster is not reported in Marriage et al. (2011a), because it was not an $\mathrm{S} / \mathrm{N}>3$ detection in the initial analysis. However, more recent analyses including data from $3 \mathrm{yr}$ of observations show that this cluster is now detected at $4.5 \sigma$, and it is therefore included in this study. Vanderlinde et al. (2010) report an SZE-estimated mass of $M_{500 c}=(2.97 \pm 0.89) \times 10^{14} h_{70}^{-1} M_{\odot}$, significantly lower than the dynamical mass reported here.

\footnotetext{
28 Five other clusters in this sample (El Gordo, ACT-CL J0235-5121, ACT-CL J0304-4921, ACT-CL J0559-5249, and ACT-CL J0707-5522) have now been included in the Planck SZ catalog (Planck Collaboration 2013).
} 
Table 7

Clusters in the Optical Program Not Detected by ACT in the 2008 Observing Season

\begin{tabular}{|c|c|c|c|c|c|}
\hline Name & $N_{\text {gal }}$ & $z_{\mathrm{BI}}$ & $\begin{array}{c}S_{\mathrm{BI}} \\
\left(\mathrm{km} \mathrm{s}^{-1}\right)\end{array}$ & $\begin{array}{c}r_{200 c} \\
\left(h_{70}^{-1} \mathrm{kpc}\right)\end{array}$ & $\begin{array}{c}M_{200 c} \\
\left(10^{14} h_{70}^{-1} M_{\odot}\right)\end{array}$ \\
\hline SCSO J0514-5126 & 15 & $0.7372 \pm 0.0018$ & $931 \pm 154$ & $1370 \pm 218$ & $6.3 \pm 3.0$ \\
\hline SCSO J0514-5140 & 22 & $0.7362 \pm 0.0011$ & $701 \pm 125$ & $1036 \pm 182$ & $2.7 \pm 1.4$ \\
\hline SCSO J0540-5614 & 17 & $0.4477 \pm 0.0008$ & $578 \pm 115$ & $990 \pm 193$ & $1.7 \pm 1.0$ \\
\hline
\end{tabular}

\subsubsection{ACT-CL J0528-5259}

This cluster was also reported by Staniszewski et al. (2009) and characterized optically by Menanteau \& Hughes (2009). Its SZE-estimated mass is $M_{500 c}=(2.9 \pm 0.9) \times 10^{14} h_{70}^{-1} M_{\odot}$ (Williamson et al. 2011) and its X-ray-estimated mass is $M_{500 c}=(3.0 \pm 0.9) \times 10^{14} h_{70}^{-1} M_{\odot}$ (Andersson et al. 2011). These values are consistent with our dynamical estimate. Also consistent with the present finding, Andersson et al. (2011) found that the X-ray morphology shows this cluster to be relaxed.

\subsubsection{ACT-CL J0546-5345}

This is the highest-redshift cluster of the sample, at $z=1.066$. Brodwin et al. (2010) first presented a spectroscopic study of this cluster based on 18 cluster members, which have been included in this study, plus the three emission-line galaxies not used for their mass measurement. We included Brodwin et al.'s (2010) galaxies in our spectroscopic catalog and applied the cluster membership algorithm (Section 3.2), which resulted in 48 members in total. Our mass estimate, calculated now with three times as many galaxies, is consistent both with their dynamical estimate and their best estimate, combining $\mathrm{X}$-ray, SZE, and dynamical information, which corresponds to $M_{200 c}=(7.9 \pm 0.9) \times 10^{14} h_{70}^{-1} M_{\odot}$.

\subsubsection{ACT-CL J0559-5249}

This cluster was also detected by SPT and reported in Vanderlinde et al. (2010). They report a simulation-based SZEderived mass $M_{500 c}=(5.3 \pm 1.2) \times 10^{14} h_{70}^{-1} M_{\odot}$, while Andersson et al. (2011) estimate an X-ray $Y_{X}$-derived mass of $M_{500 c}=(6.4 \pm 0.5) \times 10^{14} h_{70}^{-1} M_{\odot}$. Both these estimates are consistent with each other, and combined are consistent with the lower limit of our dynamical estimate. The ACT SZE signal is consistent with the dynamical mass (cf. Figure 4). Using X-ray data, Andersson et al. (2011) suggest that this cluster is in the process of merging, but our substructure analysis finds no evidence for substructure. These two results are not necessarily in contradiction since X-ray morphology and dynamical information are sensitive to substructure with different orientations.

\subsubsection{ACT-CL J0616-5227}

The optical imaging of this cluster by Menanteau et al. (2010a) was sufficient to provide confirmation but shallower than required to secure an adequate galaxy catalog for spectroscopic targeting. Out of 73 spectra obtained, only 18 are cluster members. Another six are foreground/background galaxies. The remaining are all late-type (mostly $M$ ) stars, which have similar colors to the cluster members. Both the SZE signal and the $\mathrm{X}$-rays argue in favor of this being a massive cluster, supporting the dynamical estimate.

\subsection{The SCS Clusters}

Of the 19 clusters observed during this program (see Table 2), only the 16 listed in Table 3 were detected by ACT. The other three clusters are listed in Table 7. These three clusters (hereafter "the SCS clusters") were discovered optically in the SCS and were included in the spectroscopic program because of their high optical richness (Menanteau et al. 2010b), along with ACTCL J0521-5104. Despite them being optically rich systems, the masses of the three SCS clusters are consistent with being below the ACT detection limit.

\section{CONCLUSIONS}

We have conducted a large spectroscopic follow-up program of clusters of galaxies discovered via the SZE by ACT in its southern sky survey (Menanteau et al. 2010a; Marriage et al. 2011a). We used $89 \mathrm{hr}$ of multi-object spectroscopic observations divided between FORS2 at VLT and GMOS at Gemini-South. With a few (3-4) hr of observation per cluster, we have been able to confirm an average of 65 members per cluster, which allowed us to: (1) obtain robust redshifts for each cluster; (2) measure velocity dispersions with errors $\lesssim 10 \%$, which translates to uncertainties of $<30 \%$ in mass estimates; and (3) determine the dynamical state of the clusters.

The cluster sample spans a redshift range $0.28<z<1.07$, with a median redshift $z=0.50$. Careful examination of possible substructure shows that $\sim 50 \%$ of the clusters in the ACT sample show signs of significant substructure, consistent with the X-ray study of SPT SZE-selected clusters (Andersson et al. 2011) and with optically and X-ray-selected local clusters. We find that the presence of emission-line galaxies within clusters, which could be associated with infalling groups, does not significantly modify the mass estimates. For this reason, emission-line galaxies have been included as members in the final samples.

Dynamical masses have been estimated from the radial velocity dispersions using the Evrard et al. (2008) simulationbased $\sigma-M_{200 c}$ scaling relation. These clusters have masses $6 \lesssim M_{200 c} \lesssim 21$ in units of $10^{14} h_{70}^{-1} M_{\odot}$, with a median mass $\sim 12 \times 10^{14} h_{70}^{-1} M_{\odot}$ in agreement with the mass distribution of the ACT sample as estimated from X-ray luminosities (Marriage et al. 2011a). These clusters rank therefore among the most massive clusters in the universe.

The scaling relation between dynamical mass and SZE signal has been studied using three estimators of the SZE: the central match-filtered SZE amplitude, $\widetilde{y_{0}}$, the central Compton parameter, $y_{0}$, and the Compton signal integrated within $r_{200 c}$, $Y_{200 c}$. In order to derive unbiased scaling relations, a simulationbased flux bias correction has been applied to the data, and the scaling relations include intrinsic evolution with redshift.

These scaling relations are summarized in Table 5 and represent the main result of this work. The intrinsic scatter in these relations is consistent with that predicted by simulations (e.g., Motl et al. 2005; Reid \& Spergel 2006). We find that 
Table 8

Spectroscopic Members of the 16 ACT Clusters

\begin{tabular}{|c|c|c|c|c|c|c|}
\hline Identification & $\begin{array}{c}\text { R.A. } \\
\text { (hh:mm:ss) }\end{array}$ & $\begin{array}{c}\text { Decl. } \\
\text { (dd:mm:ss) }\end{array}$ & $m_{i}$ & $z$ & $r_{c c}$ & $\begin{array}{c}\text { Main Spectral } \\
\text { Features }\end{array}$ \\
\hline SMH_J010257.7-491619.2 & $01: 02: 57.74$ & $-49: 16: 19.2$ & 19.186 & $0.87014 \pm 0.00030$ & 3.39 & $\mathrm{Ca}$ II K,H; [O II] \\
\hline SMH_J021512.3-521225.3 & $02: 15: 12.26$ & $-52: 12: 25.3$ & 18.678 & $0.48587 \pm 0.00016$ & 3.90 & $\mathrm{Ca}$ II K,H \\
\hline SMH_J023242.8-525722.3 & $02: 32: 42.80$ & $-52: 57: 22.3$ & 18.410 & $0.55592 \pm 0.00014$ & 4.53 & $\mathrm{Ca}$ II K,H \\
\hline SMH_J023545.3-512105.2 & $02: 35: 45.28$ & $-51: 21: 05.2$ & 16.493 & $0.27825 \pm 0.00015$ & 7.18 & $\mathrm{Ca}$ II K,H \\
\hline SMH_J023701.7-493810.0 & 02:37:01.71 & $-49: 38: 10.0$ & 17.582 & $0.33554 \pm 0.00016$ & 10.42 & $\mathrm{Ca}$ II K,H \\
\hline SMH_J030416.0-492126.3 & 03:04:16.04 & $-49: 21: 26.3$ & 17.463 & $0.39289 \pm 0.00020$ & 9.43 & $\mathrm{Ca}$ II K,H \\
\hline SMH_J033056.8-522813.7 & $03: 30: 56.83$ & $-52: 28: 13.6$ & 17.520 & $0.43969 \pm 0.00019$ & 10.23 & $\mathrm{Ca}$ II K,H \\
\hline SMH_J034655.5-543854.8 & $03: 46: 55.49$ & $-54: 38: 54.8$ & 18.577 & $0.53107 \pm 0.00013$ & 6.16 & Ca II K,H \\
\hline SMH_J043817.7-541920.7 & $04: 38: 17.70$ & $-54: 19: 20.6$ & 17.470 & $0.41955 \pm 0.00012$ & 9.42 & $\mathrm{Ca}$ II K,H \\
\hline SMH_J050921.3-534212.2 & $05: 09: 21.38$ & $-53: 42: 12.2$ & 18.361 & $0.46257 \pm 0.00022$ & 7.53 & Ca II K,H; [O II] \\
\hline SMH_J052114.5-510418.5 & $05: 21: 14.54$ & $-51: 04: 18.6$ & 19.060 & $0.67780 \pm 0.00041$ & 3.96 & $\mathrm{Ca}$ II K,H \\
\hline SMH_J052805.3-525952.8 & 05:28:05.30 & $-52: 59: 52.8$ & 19.715 & $0.76695 \pm 0.00037$ & 6.10 & $\mathrm{Ca}$ II K,H \\
\hline SMH_J054637.6-534531.3 & $05: 46: 37.67$ & $-53: 45: 31.3$ & 21.184 & $1.06255 \pm 0.00016$ & 6.47 & $\mathrm{Ca}$ II K,H \\
\hline SMH_J055943.2-524927.1 & $05: 59: 43.23$ & $-52: 49: 27.1$ & 19.103 & $0.61035 \pm 0.00027$ & 3.88 & $\mathrm{Ca}$ II K,H \\
\hline SMH_J061634.1-522709.9 & $06: 16: 34.05$ & $-52: 27: 09.9$ & 18.594 & $0.68765 \pm 0.00011$ & 6.87 & $\mathrm{Ca}$ II K,H \\
\hline SMH_J070704.7-552308.4 & 07:07:04.67 & $-55: 23: 08.4$ & 16.754 & $0.29451 \pm 0.00019$ & 6.05 & $\mathrm{Ca}$ II K,H \\
\hline
\end{tabular}

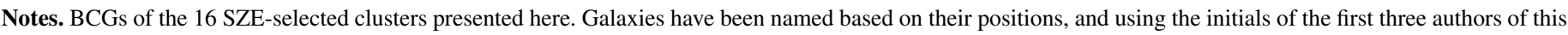
paper to identify the catalog.

(This table is available in its entirety in a machine-readable form in the online journal. A portion is shown here for guidance regarding its form and content.)

all our SZE estimators are similarly robust as mass proxies, with lognormal intrinsic scatters $\sim 15 \%$, although in the case of $Y_{200 c}-M_{200 c}$, the scatter is high but poorly constrained because of correlations between the observables. The derived scaling relations agree with the expectations from self-similar evolution of clusters. Although there are hints that dynamically disturbed clusters may bias the scaling relations, the present sample size does not allow for a robust constraint on this effect. The scaling relation between $Y_{200 c}$ and dynamical mass $M_{200 c}$ is in good agreement with previous results, which are based on different mass proxies, and predictions from simulations.

In summary, the first sample of spectroscopically followedup SZE-selected clusters from ACT has yielded results that agree with the expectations for the first generation of SZE surveys. The scaling relations derived from this sample also agree with the expectations. The results presented here show that dynamical masses provide a good way of relating the SZE to cluster masses, and larger cluster samples, in combination with other mass proxies, will serve as a tight constraint for cosmology.

We are grateful to Andrea Biviano for lengthy discussions on the corrections to the velocity dispersion, to Gary Mamon for kindly providing the velocity dispersion profiles obtained from their simulations, to Gus Evrard for helpful discussions on the aperture correction, and to Gabriel Pratt and Gilbert Holder for useful comments on the original draft. We also thank the anonymous referee for a very insightful revision of the successive versions of the draft, which helped improve the consistency and robustness of this work.

ACT operates in the Parque Astronómico Atacama in northern Chile under the auspices of Programa de Astronomía de la Comisión Nacional de Investigación Científica y Tecnológica de Chile (CONICYT). This work was supported by the U.S. National Science Foundation through awards AST-0408698 and AST-0965625 for the ACT project, and PHY-0855887, PHY-1214379, AST-0707731, and PIRE-0507768 (award No. OISE-0530095). Funding was also provided by Princeton
University, the University of Pennsylvania, and a Canada Foundation for Innovation (CFI) award to UBC. Computations were performed on the GPC supercomputer at the SciNet HPC Consortium. SciNet is funded by the CFI under the auspices of Compute Canada, the Government of Ontario, the Ontario Research Fund-Research Excellence; and the University of Toronto. This research is partially funded by "Centro de Astrofísica FONDAP" 15010003, Centro BASAL-CATA, by FONDECYT under projects 1120676 and 1085286 and by ALMA-CONICYT under projects 31090002 and 31100003.

\section{APPENDIX}

\section{ELECTRONIC DATA}

Table 8 lists the properties of the BCGs for each of the ACT clusters (see Table 3 ). This table is an excerpt from the full table available online (from which the BCGs are shown for convenience), which contains all cluster members for the 16 ACT clusters. It is given for guidance in its form and content. Column 1 lists the adopted identification, based on the J2000.0 position of each galaxy and using the initials of the first three authors of this paper to identify the catalog. Columns 2 and 3 list the positions of the galaxies. Column 4 lists the magnitude in the $i$ band, and Column 5 lists the cross-correlation redshifts and their associated errors as given by RVSAO. Column 6 lists the cross-correlation $\mathrm{S} / \mathrm{N} r_{c c}$ (Tonry \& Davis 1979), and Column 7 lists the main spectral features of each galaxy.

\section{REFERENCES}

Abazajian, K. N., Adelman-McCarthy, J. K., Ageros, M. A., et al. 2009, ApJ, 182,543

Aguerri, J. A. L., \& Sánchez-Janssen, R. 2010, A\&A, 521, A28

Akritas, M. G., \& Bershady, M. A. 1996, ApJ, 470, 706

Andersson, K., Benson, B. A., Ade, P. A. R., et al. 2011, ApJ, 738, 48

Appenzeller, I., Fricke, K., Frtig, W., et al. 1998, Msngr, 94, 1

Arnaud, M., Pratt, G. W., Piffaretti, R., et al. 2010, A\&A, 517, A92

Ashfordi, N. 2008, ApJ, 686, 201

Bahcall, N. A., \& Fan, X. 1998, ApJ, 504, 1

Balogh, M. L., Morris, S. L., Yee, H. K. C., Carlberg, R. C., \& Ellingson, E. 1999, ApJ, 527, 54 
Battaglia, N., Bond, J. R., Pfrommer, C., \& Sievers, J. L. 2012, ApJ, 758, 74 Battye, R. A., \& Weller, J. 2003, PhRvD, 68, 083506

Becker, M. R., \& Kravtsov, A. V. 2011, ApJ, 740, 25

Beers, T. C., Flynn, K., \& Gebhardt, K. 1990, AJ, 100, 32

Beers, T. C., Forman, W., Huchra, J. P., Jones, C., \& Gebhardt, K. 1991, AJ, 102,1581

Benson, B. A., Church, S. E., Ade, P. A. R., et al. 2004, ApJ, 617, 829

Benson, B. A., de Haan, T., Dudley, J. P., et al. 2013, ApJ, 763, 147

Birkinshaw, M. 1999, PhR, 310, 97

Birkinshaw, M., Hughes, J. P., \& Arnaud, K. A. 1991, ApJ, 379, 466

Biviano, A., \& Girardi, M. 2003, ApJ, 585, 205

Biviano, A., \& Katgert, P. 2004, A\&A, 424, 779

Biviano, A., Murante, G., Borgani, S., et al. 2006, A\&A, 456, 23

Bode, P., Ostriker, J. P., Cen, R., \& Trac, H. 2012, arXiv:1204.1762

Bode, P., Ostriker, J. P., \& Vikhlinin, A. 2009, ApJ, 700, 989

Bonamente, M., Joy, M., LaRoque, S. J., et al. 2008, ApJ, 675, 106

Book, L. G., \& Benson, A. J. 2010, ApJ, 716, 810

Brodwin, M., Ruel, J., Ade, P. A. R., et al. 2010, ApJ, 721, 90

Butcher, H., \& Oemler, A., Jr. 1984, ApJ, 285, 426

Carlberg, R. G., Yee, H. K. C., \& Ellingson, E. 1997, ApJ, 478, 462

Carlstrom, J. E., Ade, P. A. R., Aird, K. A., et al. 2011, PASP, 123, 568

Carlstrom, J. E., Holder, G. P., \& Reese, E. D. 2002, ARA\&A, 40, 643

Colless, M., \& Hewett, P. 1987, MNRAS, 224, 453

Coziol, R., Andernach, H., Caretta, C. A., Alamo-Martínez, K. A., \& Tago, E. 2009, AJ, 137, 4795

Danese, L., De Zotti, G., \& Di Tullio, G. 1980, A\&A, 82, 322

da Silva, A. C., Kay, S. T., Liddle, A. R., \& Thomas, P. A. 2004, MNRAS, 348,1401

Dressler, A., \& Shectman, S. A. 1988, AJ, 95, 985

Duffy, A. R., Schaye, J., Kay, S. T., \& Dalla Vecchia, C. 2008, MNRAS, 390, L64

Dünner, R., Hasselfield, M., Marriage, T. A., et al. 2013, ApJ, 762, 10

Eddington, A. S. 1913, MNRAS, 73, 359

Evrard, A. E., Bialek, J., Busha, M., et al. 2008, ApJ, 672, 122

Evrard, A. E., MacFarland, T. J., Couchman, H. M. P., et al. 2002, ApJ, 573, 7

Fadda, D., Girardi, M., Giuricin, G., Mardirossian, F., \& Mezzetti, M. 1996, ApJ, 473, 670

Faltenbacher, A., \& Diemand, J. 2006, MNRAS, 369, 1698

Fowler, J. W., Acquaviva, V., Ade, P. A. R., et al. 2010, ApJ, 722, 1148

Fowler, J. W., Niemack, M. D., Dicker, S. R., et al. 2007, ApOpt, 46, 3444

Francis, M. R., Bean, R., \& Kosowsky, A. 2005, JCAP, 12, 001

Girardi, M., Escalera, E., Fadda, D., et al. 1997, ApJ, 482, 41

Girardi, M., Fadda, D., Giuricin, G., et al. 1996, ApJ, 457, 61

Girardi, M., Giuricin, G., Mardirossian, F., Mezzetti, M., \& Boschin, W. 1998, ApJ, 505, 74

Grego, L., Carlstrom, J. E., Reese, E. D., et al. 2001, ApJ, 552, 2

Hand, N., Appel, J. W., Battaglia, N., et al. 2011, ApJ, 736, 39

Hansen, S. M., Sheldon, E. S., Wechsler, R. H., \& Koester, B. P. 2009, ApJ, 699,1333

Hasselfield, M., Hilton, M., Marriage, T. A., et al. 2013, arXiv:1301.0816

Heisler, J., Tremaine, S., \& Bahcall, J. N. 1985, ApJ, 298, 8

Hincks, A. D., Acquaviva, V., Ade, P. A. R., et al. 2010, ApJS, 191, 423

High, F. W., Stalder, B., Song, J., et al. 2010, ApJ, 723, 1736

Hilton, M., Hasselfield, M., Sifón, C., et al. 2013, arXiv:1301.0780

Hook, I., Jørgensen, I., Allington-Smith, J. R., et al. 2004, PASP, 116, 425

Hughes, J. P., \& Birkinshaw, M. 1998, ApJ, 501, 1

Jenkins, A., Frenk, C. S., White, S. D. M., et al. 2001, MNRAS, 321, 372

Katgert, P., Biviano, A., \& Mazure, A. 2004, ApJ, 600, 657

Katgert, P., Mazure, A., Perea, J., et al. 1996, A\&A, 310, 8

Kelly, B. C. 2007, ApJ, 665, 1489

Komatsu, E., Smith, K. M., Dunkley, J., et al. 2011, ApJS, 192, 18

Kurtz, M. J., \& Mink, D. J. 1998, PASP, 110, 934

LaRoque, S. J., Bonamente, M., Carlstrom, J. E., et al. 2006, ApJ, 652, 917

Lin, Y.-T., \& Mohr, J. J. 2004, ApJ, 617, 879

Łokas, E. L., Wojtak, R., Gottlöber, S., Mamon, G. A., \& Prada, F. 2006, MNRAS, 367, 1463
Mamon, G. A., Biviano, A., \& Murante, G. 2010, A\&A, 520, A30

Mantz, A., Allen, S. W., Ebeling, H., Rapetti, D., \& Drlica-Wagner, A. 2010a, MNRAS, 406, 1773

Mantz, A., Allen, S. W., Rapetti, D., \& Ebeling, H. 2010b, MNRAS, 406,1759

Mantz, A., Allen, S. W., \& Rapetti, D. 2010c, MNRAS, 406, 1805

Marriage, T. A., Acquaviva, V., Ade, P. A. R., et al. 2011a, ApJ, 737, 61

Marriage, T. A., Baptiste Juin, J., Lin, Y.-T., et al. 2011b, ApJ, 731, 100

Marrone, D. P., Smith, G. P., Okabe, N., et al. 2012, ApJ, 754, 119

Marrone, D. P., Smith, G. P., Richard, J., et al. 2009, ApJL, 701, L114

Melin, J.-B., Bartlett, J. G., Delabrouille, J., et al. 2011, A\&A, 525, A139

Menanteau, F., González, J., Juin, J.-B., et al. 2010a, ApJ, 723, 1523

Menanteau, F., \& Hughes, J. P. 2009, ApJL, 694, L136

Menanteau, F., Hughes, J. P., Barrientos, L. F., et al. 2010b, ApJS, 191, 340

Menanteau, F., Hughes, J. P., Sifón, C., et al. 2012, ApJ, 748, 7

Moore, B., Lake, G., Quinn, T., \& Stadel, J. 1999, MNRAS, 304, 465

Mortonson, M. J., Hu, W., \& Huterer, D. 2011, PhRvD, 83, 023015

Motl, P. M., Hallman, E. J., Burns, J. O., \& Norman, M. L. 2005, ApJL, 623, L63

Nagai, D. 2006, ApJ, 650, 538

Navarro, J. F., Frenk, C. S., \& White, S. D. M. 1995, MNRAS, 275, 56

Oegerle, W. R., \& Hill, J. M. 2001, AJ, 122, 2858

Pimbblet, K. A., Roseboom, I. G., \& Doyle, M. T. 2006, MNRAS, 368, 651

Pinkney, J., Roettiger, K., Burns, J. O., \& Bird, C. M. 1996, ApJS, 104, 36

Planck Collaboration 2011a, A\&A, 536, A8

Planck Collaboration 2011b, A\&A, 536, A11

Planck Collaboration 2011c, A\&A, 536, A12

Planck Collaboration 2011d, A\&A, 536, A9

Planck Collaboration 2013, arXiv:1303.5089

Pratt, G. W., Croston, J. H., Arnaud, M., \& Böhringer, H. 2009, A\&A, 498, 361

Quintana, H., Carrasco, E. R., \& Reisenegger, A. 2000, AJ, 120, 511

Quintana, H., \& Lawrie, D. G. 1982, AJ, 87, 1

Reid, B. A., \& Spergel, D. N. 2006, ApJ, 651, 643

Rines, K., Geller, M. J., \& Diaferio, A. 2010, ApJL, 715, L180

Rines, K., Geller, M. J., Kurtz, M. J., \& Diaferio, A. 2003, AJ, 126, 2152

Rozo, E., Wechsler, R. H., Rykoff, E. S., et al. 2010, ApJ, 708, 645

Schuecker, P., Böhringer, H., Reiprich, T. H., \& Feretti, L. 2001, A\&A, 378, 408

Sealfon, C., Verde, L., \& Jimenez, R. 2006, ApJ, 649, 118

Sehgal, N., Bode, P., Das, S., et al. 2010, ApJ, 709, 920

Sehgal, N., Trac, H., Acquaviva, V., et al. 2011, ApJ, 732, 44

Shaw, L. D., Holder, G. P., \& Bode, P. 2008, ApJ, 686, 206

Skibba, R. A., van den Bosch, F. C., Yang, X., et al. 2011, MNRAS, 410,417

Springel, V., White, M., \& Hernquist, L. 2001a, ApJ, 549, 681

Springel, V., White, S. D. M., Tormen, G., \& Kauffmann, G. 2001b, MNRAS, 328,726

Staniszewski, Z., Ade, P. A. R., Aird, K. A., et al. 2009, ApJ, 701, 32

Sunyaev, R. A., \& Zel'dovich, Y. B. 1970, Ap\&SS, 7, 20

Sunyaev, R. A., \& Zel'dovich, Y. B. 1980, MNRAS, 190, 413

Swetz, D. S., Ade, P. A. R., Amiri, M., et al. 2011, ApJS, 194, 41

Tauber, J. A., Mandolesi, N., Puget, J.-L., et al. 2010, A\&A, 520, A1

Teerikorpi, P. 1997, ARA\&A, 35, 101

The, L. S., \& White, S. D. M. 1986, AJ, 92, 1248

Tinker, J., Kravtsov, A. V., Klypin, A., et al. 2008, ApJ, 688, 709

Tonry, J., \& Davis, M. 1979, AJ, 84, 1511

Umetsu, K., Birkinshaw, M., Liu, G.-C., et al. 2009, ApJ, 694, 1643

Vanderlinde, K., Crawford, T. M., de Haan, T., et al. 2010, ApJ, 722, 1180

van Dokkum, P. 2001, PASP, 113, 1420

Vikhlinin, A., Kravtsov, A. V., Burenin, R. A., et al. 2009, ApJ, 692, 1060

Voit, G. M. 2005, RvMP, 77, 207

Werner, N., Churazov, E., Finoguenov, A., et al. 2007, A\&A, 474, 707

Williamson, R., Benson, B. A., High, F. W., et al. 2011, ApJ, 738, 139

Yang, H.-Y. K., Bhattacharya, S., \& Ricker, P. M. 2010, ApJ, 725, 1124

Zel'dovich, Y. B., \& Sunyaev, R. A. 1969, Ap\&SS, 4, 301 